\title{
SECOND ORDER NECESSARY CONDITIONS FOR OPTIMAL CONTROL PROBLEMS OF EVOLUTION EQUATIONS INVOLVING FINAL POINT EQUALITY CONSTRAINTS
}

\author{
HÉLÈne Frankowska ${ }^{1, *, * *}$ AND QI Lü̈2,***
}

\begin{abstract}
We establish some second order necessary conditions for optimal control problems of evolution equations involving final point equality and inequality constraints. Compared with the existing works, the main difference is due to the presence of end-point equality constraints. With such constraints, we cannot simply use the variational techniques since perturbations of a given control may be no longer admissible. We also cannot use the Ekeland's variational principle, which is a first order variational principle, to obtain second order necessary conditions. Instead, we combine some inverse mapping theorems on metric spaces and second order linearization of data to obtain our results.
\end{abstract}

Mathematics Subject Classification. 49K20.

Received October 9, 2020. Accepted June 12, 2021.

\section{INTRODUCTION}

Let $H$ be a separable Hilbert space and $A: D(A) \rightarrow H$ an infinitesimal generator of a $C_{0}$-semigroup $\left\{e^{A t}\right\}_{t \geq 0}$ on $H$. Let $H_{1}$ be a separable Banach space and $U \subset H_{1}$ be a nonempty bounded closed subset. Let $T>0$. Put

$$
\mathcal{U} \triangleq\{u:[0, T] \rightarrow U \mid u(\cdot) \text { is Lebesgue measurable }\}
$$

and define a distance on $\mathcal{U}$ as follows:

$$
\mathbf{d}\left(u_{1}, u_{2}\right) \triangleq\left|u_{1}-u_{2}\right|_{L^{1}\left(0, T ; H_{1}\right)}, \quad \forall u_{1}, u_{2} \in \mathcal{U}
$$

Then $(\mathcal{U}, \mathbf{d})$ is a complete separable metric space.

** The research of this author is partially supported by the AFOSR grant FA 9550-18-1-0254 and the CNRS-NSFC PRC Project under grant 271392. She also benefited from the support of the FJMH Program PGMO and from the support to this program from EDF-THALES-ORANGE-CRITEO.

*** The research of this author is supported by the NSF of China under grants 11971334, 12025105 and 11931011, by the Chang Jiang Scholars Program from the Chinese Education Ministry, and the CNRS-NSFC PRC Project under grant 11530142.

Keywords and phrases: Optimal control, time evolution partial differential equation, second order necessary condition, local minimizer.

1 CNRS, IMJ-PRG, UMR 7586, Sorbonne Université, case 247, 4 place Jussieu, 75252 Paris, France.

2 School of Mathematics, Sichuan University, Chengdu, 610064, PR China.

* Corresponding author: helene.frankowska@imj-prg.fr 
Consider the following control system:

$$
\left\{\begin{array}{l}
x_{t}(t)=A x(t)+f(t, x(t), u(t)), \quad t \in(0, T], \\
x(0)=x_{0}, u \in \mathcal{U},
\end{array}\right.
$$

where $x_{0} \in H$ is given and the map $f:[0, T] \times H \times H_{1} \rightarrow H$ satisfies the following conditions:

(H1) For all $(x, u) \in H \times H_{1}, f(\cdot, x, u)$ is Lebesgue measurable, for all $(t, x) \in[0, T] \times H, f(t, x, \cdot)$ is continuous and there exists $C>0$ such that

$$
\sup _{u \in U}|f(t, x, u)|_{H} \leq C\left(1+|x|_{H}\right), \quad \forall(t, x) \in[0, T] \times H .
$$

(H2) For every $r>0$, there exists $k_{r}(\cdot) \in L^{1}(0, T)$ such that for a.e. $t \in[0, T]$

$$
\left|f\left(t, x_{1}, u\right)-f\left(t, x_{2}, u\right)\right|_{H} \leq k_{r}(t)\left|x_{1}-x_{2}\right|_{H}
$$

for any $x_{1}, x_{2} \in H$ with $\left|x_{1}\right|_{H} \leq r,\left|x_{2}\right|_{H} \leq r$ and all $u \in U$.

Here and in what follows, $C$ denotes a generic constant which may change from line to line.

Under the assumptions (H1) and (H2), to each $u \in \mathcal{U}$ there corresponds a unique (mild) solution $x \in$ $C([0, T] ; H)$ of the system (1.1) called a trajectory of (1.1).

Let $r \in \mathbb{N}, g_{j} \in C(H ; \mathbb{R})$ for $j=1, \ldots, r, H_{2}$ be a separable Hilbert space and $h \in C\left(H ; H_{2}\right)$. We consider the following final state constraints

$$
g_{j}(x(T)) \leq 0, \quad j=1, \ldots, r
$$

and

$$
h(x(T))=0 .
$$

Let $g_{0} \in C(H ; \mathbb{R})$ and consider the following Mayer type cost functional:

$$
J(u)=g_{0}(x(T)),
$$

where $x(\cdot)$ is the solution to (1.1) corresponding to the control $u(\cdot)$ such that (1.3) and (1.4) hold.

Any trajectory-control pair $(x, u)$ of (1.1) satisfying (1.3) and (1.4) is called an admissible pair. The corresponding $x$ and $u$ are called admissible trajectory and admissible control, respectively.

Definition 1.1. An admissible pair $(\bar{x}, \bar{u})$ is called a local minimizer of (1.5) if for some $\varepsilon>0$, we have $g_{0}(x(T)) \geq g_{0}(\bar{x}(T))$ for each admissible trajectory-control pair $(x, u)$ such that $|u-\bar{u}|_{L^{1}\left(0, T ; H_{1}\right)}<\varepsilon$.

It is one of the important issues in optimal control theory to characterize local minimizers. Similarly to the Calculus, the most usual way to do this is to find necessary conditions satisfied by local minimizers. Since the milestone in [17], first-order necessary conditions are studied extensively in the literature for different kinds of control systems, such as systems governed by ordinary differential equations (e.g. [17]), systems governed by partial differential equations (e.g. [13]), systems governed by stochastic differential equations (e.g. [21]), systems governed by stochastic evolution equations (e.g. [15]), etc.

Second order necessary conditions for controlled partial differential equations are studied extensively in the literature (e.g. $[3-5,18-20])$. However, as far as we know, there are no published results on second order necessary conditions with end-point equality constraints. The main purpose of this paper is to investigate such kind of 
problems. Second order necessary conditions have the same purpose as the first order conditions - to eliminate some candidates for optimality that the first order conditions did not throw out. Sufficient conditions are usually obtained by strengthening the obtained second order necessary conditions. This is planned as a future direction of our research.

The main difficulties we face are as follows:

- The interior of the tangent space to constraints defined by equalities is, in general, empty. Then one cannot simply use the variational technique to obtain second order necessary conditions since perturbations of the optimal control may be not admissible.

- Unlike for the first order necessary condition, it is unclear whether one can use the Ekeland variational principle, which is a first order variational principle, to derive second order necessary conditions.

- Unlike in the finite dimensional case, in the Hilbert spaces, in general, two convex sets having an empty intersection can not be separated by a linear functional: at least one of them has to have interior points. When there are several constraints, this requires all but one of them to have a nonempty interior. In the presence of the end point equality constraints we face two sets with empty interiors, the second one coming from the control system itself, that is usually seen as a second equality constraint.

In the literature these difficulties are usually handled by reducing the optimal control problem to an abstract infinite dimensional mathematical programming one. For this aim it is usually requested that control sets are described via inequality and equality constraints verifying some gradients independence assumptions. In particular, if the set of controls is a union of such sets, instead of their intersection, such methodology does not work anymore. In contrast, in our paper the control set $U$ is an arbitrary nonempty closed subset of a Banach space.

Usually, once such new abstract optimisation problem is stated, some abstract constraints qualification assumptions (Robinson's like conditions) allow to write a Lagrange multiplier rule. The main difficulty is then to return back to the original problem and to translate the derived multiplier rule in terms of the original problem. For this reason very often the authors restrict their attention to very simple sets $U$, impose some structural assumptions on optimal controls and assume that optimal controls are piecewise continuous. Such assumptions are very strong, because usually, in the presence of constraints, the classical existence results guarantee only measurability of optimal controls with no particular structure.

In the difference with this approach, we do not make reduction of the optimal control of evolution system to an abstract mathematical programming problem. Instead we linearize twice the control system and the constraints in the original state space and apply the separation theorems to the linearized problem. This allows us to work with measurable controls by the methods of variational analysis. Another advantage of our approach is that it brings also sufficient conditions for the normality of the derived necessary conditions.

To overcome the difficulties mentioned above, we borrow some ideas from [8], where an optimal control problem was investigated in the finite dimensional setting. Let us underline that usually the second order tangents to reachable sets of control systems do have an empty interior. Same for the second order tangents to the sets defined by the equality constraints. The separation theorems can not be applied then in the usual way to get second order conditions like in [10,12]. We exploit instead the idea from [8] saying that under a surjectivity type assumption imposed on the linearized control system, the intersection of the two second order tangents is the second order tangent to the reachable set of the control system with a final point equality constraint. For this aim a non-standard inverse mapping theorem on a metric space is applied. We remain then with just one set having an empty interior and the application of the separation theorem becomes then possible. Roughly speaking, the inverse mapping theorem on a metric space allows us to consider equality constraints as a part of control system because its second order linearization incorporates the second order linearization of the end-point equality constraints. More details can be found in Section 4.

The rest of this paper is organized as follows: in Section 2, we present our main results, whose proofs are given in Section 4. Section 3 is devoted to some preliminaries. At last, in Section 5, we provide two illustrative examples. 


\section{Statement of the MAin RESUlts}

To begin with, let us introduce some notations. Let $Z$ be a Banach space with the norm $|\cdot|_{Z}$. For any subset $\mathcal{K} \subset Z$, denote by int $\mathcal{K}, \mathrm{cl} \mathcal{K}$ and $\partial \mathcal{K}$ the interior, closure and boundary of $\mathcal{K}$, respectively. A subset $\mathcal{K}$ is called a cone if $\alpha z \in \mathcal{K}$ for every $\alpha \geq 0$ and $z \in \mathcal{K}$. For a general $\mathcal{K}$, denote by $\overline{c o} \mathcal{K}$ the smallest closed convex set containing $\mathcal{K}$. Define the distance between a point $z \in Z$ and $\mathcal{K}$ as

$$
\operatorname{dist}(z, \mathcal{K}) \triangleq \inf _{y \in \mathcal{K}}|y-z|_{Z}
$$

Definition 2.1. For $z \in \mathcal{K}$, the Clarke tangent cone $\mathcal{C}_{\mathcal{K}}(z)$ to $\mathcal{K}$ at $z$ is

$$
\mathcal{C}_{\mathcal{K}}(z) \triangleq\left\{v \in Z \mid \lim _{\varepsilon \rightarrow 0^{+}, y \in \mathcal{K}, y \rightarrow z} \frac{\operatorname{dist}(y+\varepsilon v, \mathcal{K})}{\varepsilon}=0\right\},
$$

and the adjacent cone $T_{\mathcal{K}}^{b}(z)$ to $\mathcal{K}$ at $z$ is

$$
T_{\mathcal{K}}^{b}(z) \triangleq\left\{v \in Z \mid \lim _{\varepsilon \rightarrow 0^{+}} \frac{\operatorname{dist}(z+\varepsilon v, \mathcal{K})}{\varepsilon}=0\right\} .
$$

$\mathcal{C}_{\mathcal{K}}(z)$ is a closed convex cone in $Z$ and $\mathcal{C}_{\mathcal{K}}(z) \subset T_{\mathcal{K}}^{b}(z)$. When $\mathcal{K}$ is convex, $\mathcal{C}_{\mathcal{K}}(z)=T_{\mathcal{K}}^{b}(z)=\operatorname{cl}\{\alpha(\hat{z}-z) \mid \alpha \geq$ $0, \hat{z} \in \mathcal{K}\}$.

Definition 2.2. For $z \in \mathcal{K}$ and $v \in Z$, the second order adjacent subset to $\mathcal{K}$ at $(z, v)$ is

$$
T_{\mathcal{K}}^{b(2)}(z, v) \triangleq\left\{h \in Z \mid \lim _{\varepsilon \rightarrow 0^{+}} \frac{\operatorname{dist}\left(z+\varepsilon v+\varepsilon^{2} h, \mathcal{K}\right)}{\varepsilon^{2}}=0\right\}
$$

The set $T_{\mathcal{K}}^{b(2)}(z, v)$ is closed and may be empty for instance when $v \notin T_{\mathcal{K}}^{b}(z)$.

Let $Y_{1}, Y_{2}$ and $Z$ be Banach spaces. Denote by $\mathcal{L}\left(Y_{1} ; Z\right)$ the Banach space of all bounded linear operators from $Y_{1}$ to $Z$ and by $\mathcal{L}\left(Y_{1}, Y_{2} ; Z\right)$ the Banach space of all bounded bilinear operators from $Y_{1} \times Y_{2}$ to $Z$. When $Y_{1}=Z$, we replace $\mathcal{L}\left(Y_{1} ; Z\right)$ by $\mathcal{L}\left(Y_{1}\right)$ for simplicity.

The Hamiltonian and the terminal Lagrange function are defined respectively by

$$
\mathcal{H}(t, x, u, p)=\langle p, f(t, x, u)\rangle_{H}
$$

and

$$
l(x, \alpha, \beta)=\sum_{j=0}^{r} \alpha_{j} g_{j}(x)+\langle\beta, h(x)\rangle_{H_{2}},
$$

where $\alpha=\left(\alpha_{0}, \alpha_{1}, \cdots, \alpha_{r}\right) \in \mathbb{R}_{+}^{r+1}$ and $\beta \in H_{2}$. Set

$$
K_{j} \triangleq\left\{x \in H \mid g_{j}(x) \leq 0\right\} \text { for } j=1, \ldots, r .
$$

Clearly, for every $x \in \partial K_{j}$, we have $g_{j}(x)=0$.

Let $(\bar{x}, \bar{u})$ be a local minimizer of (1.5). Put

$$
\mathcal{I}_{g} \triangleq\left\{j=1, \ldots, r \mid \bar{x}(T) \in \partial K_{j}\right\}
$$


When $f(t, \cdot, \cdot), g_{j}(j=0, \ldots, r)$ and $h$ are $C^{1}$, the first-order necessary condition for local minimizers is as follows:

Theorem 2.3. Assume (H1), that the maps $f(t, \cdot, \cdot), g_{j}(j=0, \ldots, r)$ and $h$ are continuously Fréchet differentiable for a.e. $t \in[0, T]$ and

$$
\left|f_{x}(t, x, u)\right|_{\mathcal{L}(H)}+\left|f_{u}(t, x, u)\right|_{\mathcal{L}\left(H_{1} ; H\right)}+\sum_{j=0}^{r}\left|g_{j, x}(x)\right|_{H}+\left|h_{x}(x)\right|_{\mathcal{L}\left(H ; H_{2}\right)} \leq C, \quad \forall(t, x, u) \in[0, T] \times H \times U .
$$

Then there exist $\alpha=\left(\alpha_{0}, \alpha_{1}, \ldots, \alpha_{r}\right) \in \mathbb{R}_{+}^{r+1}$ and $\beta \in H_{2}$, not vanishing simultaneously, satisfying

$$
\alpha_{j}=0 \quad \text { if } \quad j \notin \mathcal{I}_{g}
$$

such that for the (mild) solution $p \in C([0, T] ; H)$ of

$$
\left\{\begin{array}{l}
-p_{t}(t)=A^{*} p(t)+\mathcal{H}_{x}(t, \bar{x}(t), \bar{u}(t), p(t)), \quad t \in[0, T), \\
p(T)=l_{x}(\bar{x}(T), \alpha, \beta),
\end{array}\right.
$$

we have

$$
\inf _{\kappa \in \mathcal{C}_{U}(\bar{u}(t))} \mathcal{H}_{u}(t, \bar{x}(t), \bar{u}(t), p(t))(\kappa) \geq 0, \quad \text { for a.e. } t \in[0, T],
$$

where $\mathcal{H}_{x}$ and $\mathcal{H}_{u}$ are the Fréchet derivatives of $\mathcal{H}$ with respect to $x$ and $u$, respectively.

When $U$ is convex and $f$ is affine with respect to $u$, Theorem 2.3 is just the Pontryagin minimum principle for the local minimizer $(\bar{x}, \bar{u})$. When $U$ is not convex, generally speaking, the conclusion of Theorem 2.3 is weaker than the Pontryagin maximum principle derived by the needle variation technique (e.g., [13]) for the so called strong local minimizers, i.e. when local means that trajectories stay nearby $\bar{x}$ (instead of controls nearby $\bar{u}$ ). Note that under our assumptions any strong local minimizer is also a weak local minimizer. However, in general the converse is not true. Thus the first order results for local minimizers, in general, can not be deduced from those known for strong local minimizers. In the recent publication [11], in the finite dimensional setting, the authors derived the second order conditions for local minimizers together with the maximum principle using a relaxation theorem. We do not have yet such analogue for the infinite dimensional context.

Theorem 2.3 can be proved by the separation theorem for convex sets. Since the main purpose of this paper is to study second order necessary conditions, we shall derive the first-order condition as a consequence of the second-order one and, so, for $C^{2}$ data.

Let us introduce the following assumption:

(H3) The maps $f(t, \cdot, \cdot), g_{j}(j=0, \ldots, r)$ and $h$ are twice continuously Fréchet differentiable for a.e. $t \in[0, T]$. Moreover, ${ }^{1}$

$$
\begin{aligned}
& \left|f_{x}(t, x, u)\right|_{\mathcal{L}(H)}+\left|f_{x x}(t, x, u)\right|_{\mathcal{L}(H, H ; H)}+\left|f_{x u}(t, x, u)\right|_{\mathcal{L}\left(H, H_{1} ; H\right)} \\
& +\left|f_{u}(t, x, u)\right|_{\mathcal{L}\left(H_{1} ; H\right)}+\left|f_{u u}(t, x, u)\right|_{\mathcal{L}\left(H_{1}, H_{1} ; H\right)} \leq C, \quad \forall(t, x, u) \in[0, T] \times H \times U
\end{aligned}
$$

\footnotetext{
${ }^{1}$ Recall that, for Banach spaces $Y$ and $Z$, a $C^{2}$-function $\varphi: Y \rightarrow Z$ and any $\eta \in Y, \varphi_{x x}(\eta) \in \mathcal{L}(Y, Y$; $Z)$. This means that, for any $\eta_{1}, \eta_{2} \in Y, \varphi_{x x}(\eta)\left(\eta_{1}, \eta_{2}\right) \in Z$.
} 
and

$$
\sum_{j=0}^{r}\left(\left|g_{j, x}(x)\right|_{H}+\left|g_{j, x x}(x)\right|_{\mathcal{L}(H, H ; \mathbb{R})}\right)+\left|h_{x}(x)\right|_{\mathcal{L}\left(H ; H_{2}\right)}+\left|h_{x x}(x)\right|_{\mathcal{L}\left(H, H ; H_{2}\right)} \leq C, \quad \forall x \in H .
$$

Now, let us introduce two variational linearizations of control system $(1.1)$ at $(\bar{x}, \bar{u})$. The first one is

$$
\left\{\begin{array}{l}
y_{t}(t)=A y(t)+f_{x}[t] y(t)+f_{u}[t] u(t), u(t) \in T_{U}^{b}(\bar{u}(t)), \quad t \in(0, T], \\
y(0)=0, u \in L^{1}\left(0, T ; H_{1}\right),
\end{array}\right.
$$

where $f_{x}[t] \triangleq f_{x}(t, \bar{x}(t), \bar{u}(t))$ and $f_{u}[t] \triangleq f_{u}(t, \bar{x}(t), \bar{u}(t))$.

The second one is

$$
\left\{\begin{array}{l}
\tilde{y}_{t}(t)=A \tilde{y}(t)+f_{x}[t] \tilde{y}(t)+v(t), v(t) \in \overline{\mathrm{co}} f(t, \bar{x}(t), U)-f[t], \quad t \in(0, T], \\
\tilde{y}(0)=0
\end{array}\right.
$$

where $v:[0, T] \rightarrow H_{1}$ is measurable and $f[t] \triangleq f(t, \bar{x}(t), \bar{u}(t))$.

The reachable set of (2.7) at time $T$ is

$$
R^{L}=\{\tilde{y}(T) \mid \tilde{y}(\cdot) \text { is a trajectory of }(2.7)\} .
$$

Clearly, $R^{L}$ is convex. Put

$$
\Xi \triangleq C([0, T] ; H) \times L^{2}\left(0, T ; H_{1}\right)
$$

To study second order conditions we introduce the $\operatorname{set} \mathcal{C}(\bar{x}, \bar{u})$ of all critical pairs $(y, u) \in \Xi$ solving the linear system (2.6) such that

$$
\begin{gathered}
g_{0, x}(\bar{x}(T))(y(T)) \leq 0, \\
h_{x}(\bar{x}(T))(y(T))=0, \\
g_{j, x}(\bar{x}(T))(y(T)) \leq 0, \quad \text { for } j \in \mathcal{I}_{g},
\end{gathered}
$$

and

$$
\exists \delta_{0}>0, \exists c \in L^{2}(0, T), \forall \delta \in\left[0, \delta_{0}\right], \operatorname{dist}(\bar{u}(t)+\delta u(t), U) \leq c(t) \delta^{2} \text { for a.e. } t \in[0, T] .
$$

The last inequality strengthens the inclusion $u(t) \in T_{U}^{b}(\bar{u}(t))$ and is very useful in the second order analysis involving such stronger tangents. Thus the critical set $\mathcal{C}(\bar{x}, \bar{u})$ can be seen as the set of all the solutions to the strengthened linearized system (2.6) that satisfy the linearized final point constraints $(2.9),(2.10)$ and is critical in the sense of (2.8). Our proofs below imply that for any $(y, u) \in \mathcal{C}(\bar{x}, \bar{u})$ we have $g_{0, x}(\bar{x}(T))(y(T))=0$ and, consequently, the word critical is inherited from the classical Calculus. 
Put

$$
\Lambda(\bar{x}, \bar{u}) \triangleq\left\{(\alpha, \beta, p) \in \mathbb{R}_{+}^{r+1} \times H_{2} \times C([0, T] ; H) \mid(\alpha, \beta, p) \neq 0 \text { and satisfies }(2.1)-(2.3)\right\} .
$$

For any $(\alpha, \beta, p) \in \Lambda(\bar{x}, \bar{u}), u:[0, T] \rightarrow H_{1}$ and $t \in[0, T]$, define

$$
\Upsilon(u(t), p(t)) \triangleq \inf \left\{\mathcal{H}_{u}[t](v) \mid v \in T_{U}^{b(2)}(\bar{u}(t), u(t))\right\},
$$

where $\mathcal{H}_{u}[t]=\mathcal{H}_{u}(t, \bar{x}(t), \bar{u}(t), p(t))$, and by convention, $\inf \emptyset=+\infty$.

For every $(\alpha, \beta, p) \in \Lambda(\bar{x}, \bar{u})$ and $(y, u) \in \Xi$, define

$$
\begin{aligned}
\Omega(y, u, \alpha, \beta, p) \triangleq & l_{x x}(\bar{x}(T), \alpha, \beta)(y(T), y(T)) \\
& +\int_{0}^{T}\left(\mathcal{H}_{x x}[t](y(t), y(t))+2 \mathcal{H}_{x u}[t](y(t), u(t))+\mathcal{H}_{u u}[t](u(t), u(t))\right) \mathrm{d} t,
\end{aligned}
$$

where $\mathcal{H}_{x x}[t]=\mathcal{H}_{x x}(t, \bar{x}(t), \bar{u}(t), p(t))$ and $\mathcal{H}_{x u}[t], \mathcal{H}_{u u}[t]$ are defined in a similar way.

Fix a trajectory-control pair $(y, u) \in \Xi$ of $(2.6)$. We introduce a second order linearization

$$
\left\{\begin{aligned}
w_{t}(t)= & A w+f_{x}[t] w(t)+f_{u}[t] v(t)+\frac{1}{2}\left[f_{x x}[t](y(t), y(t))\right. \\
& \left.+2 f_{x u}[t](y(t), u(t))+f_{u u}[t](u(t), u(t))\right], v(t) \in T_{U}^{b(2)}(\bar{u}(t), u(t)), \text { a.e. } t \in(0, T], \\
w(0)= & 0, v \in L^{1}\left(0, T ; H_{1}\right),
\end{aligned}\right.
$$

where $f_{x x}[t]=f_{x x}(t, \bar{x}(t), \bar{u}(t))$ and $f_{x u}[t], f_{u u}[t]$ are similarly defined. Denote by $R^{L(2)}$ the reachable set at time $T$ of $(2.13)$, which depends on the choice of $(y, u)$. It is well known that the set $\operatorname{cl} R^{L(2)}$ is convex. This can be easily deduced from Corollary 3.9 below.

Define the convex sets

$$
\Theta=\left\{\theta \in H \mid h_{x}(\bar{x}(T)) \theta+\frac{1}{2} h_{x x}(\bar{x}(T))(y(T), y(T))=0\right\}
$$

and

$$
\widetilde{\Theta}=\left\{\theta-\kappa \mid \theta \in \Theta, \kappa \in \operatorname{cl}\left(R^{L(2)}\right)\right\} .
$$

Clearly, both $\Theta$ and $\widetilde{\Theta}$ depend on the choice of $(y, u)$.

We impose the following additional assumption.

(H4) There is a closed subspace $\widetilde{H}$ of $H$ such that $\widetilde{\Theta} \subset \widetilde{H}$ and $\operatorname{int}_{\widetilde{H}} \widetilde{\Theta} \neq \emptyset$, where $\operatorname{int}_{\widetilde{H}} \widetilde{\Theta}$ denotes the interior of $\widetilde{\Theta}$ in $\widetilde{H}$.

Remark 2.4. (H4) is introduced to apply the Hahn-Banach theorem to separate two convex sets. Indeed, it is well known that to separate two convex sets in an infinite dimensional Hilbert space, one of them should have an interior point. Usually, $\widetilde{\Theta}$ does not satisfy this requirement. To remedy that difficulty, we assume that $\operatorname{int}_{\widetilde{H}} \widetilde{\Theta} \neq \emptyset$. Under this condition, for any nonempty convex subset $K$ of $\widetilde{H}$ having an empty intersections with $\widetilde{\Theta}$ one can find a linear functional to separate $\widetilde{\Theta}$ from $K$. More details can be found in the proof of Theorem 2.5. 
For any given $u:[0, T] \rightarrow H_{1}$, set

$$
\begin{array}{r}
V^{2}(\bar{u}, u) \triangleq\left\{v:[0, T] \rightarrow H_{1} \mid v \text { is measurable, } f_{u}[\cdot] v(\cdot)\right. \text { is integrable and } \\
\left.\qquad v(t) \in T_{U}^{b(2)}(\bar{u}(t), u(t)) \text { for a.e. } t \in[0, T]\right\}
\end{array}
$$

Theorem 2.5. Assume (H1), (H3) and let $(\bar{x}, \bar{u})$ be a local minimizer of (1.5) satisfying

$$
0 \in \operatorname{int} \operatorname{cl} h_{x}(\bar{x}(T))\left(R^{L}\right)
$$

and

$$
h_{x}(\bar{x}(T))(H)=H_{2}
$$

Let $(y, u) \in \mathcal{C}(\bar{x}, \bar{u})$ be such that $\left(\boldsymbol{H}_{4}\right)$ is satisfied and $V^{2}(\bar{u}, u) \cap L^{2}\left(0, T ; H_{1}\right) \neq \emptyset$. Then there exists $(\alpha, \beta, p) \in$ $\Lambda(\bar{x}, \bar{u})$ such that $\alpha_{j}=0$ whenever $g_{j, x}(\bar{x}(T))(y(T))<0$, the function $\Upsilon(u, p)$ is integrable and

$$
\frac{1}{2} \Omega(y, u, \alpha, \beta, p)+\int_{0}^{T} \Upsilon(u(t), p(t)) \mathrm{d} t \geq 0 .
$$

Remark 2.6. Condition (2.17) is an assumption on the controllability of the system (2.7). For a given controlled partial differential equation, if the optimal control belongs to the interior of $U$ for a.e. $t \in[0, T]$ and $(2.18)$ is satisfied, then one can use the classical controllability theory of partial differential equations to verify $(2.17)$ (e.g. [22]). For establishing the first order necessary condition, these assumptions can be relaxed (e.g. [13, 14]). On the other hand, if there is no state constraint (1.4), then (2.17) and (2.18) can be omitted because in this case $h$ would not be involved in our proofs. Since $R^{L}$ is convex and (2.7) is linear in $\widetilde{y},(2.17)$ can be verified by a separation theorem whenever $\operatorname{cl} h_{x}(\bar{x}(T))\left(R^{L}\right)$ has a nonempty interior.

Recall that if $\alpha_{0}>0$, then the necessary condition in Theorem 2.5 is called normal. In such case, by normalizing, one can set $\alpha_{0}=1$. On the other hand, in the abnormal case i.e. when $\alpha_{0}=0$, the necessary condition is independent of the cost functional. Hence, it is important to show the normality of the multiplier. We present a second-order sufficient condition for normality below. To this end, let us introduce the following linear control system

$$
\left\{\begin{array}{l}
z_{t}(t)=A z(t)+f_{x}[t] z(t)+f_{u}[t] \pi(t), \pi(t) \in \mathcal{C}_{U}(\bar{u}(t)), \quad t \in(0, T] \\
z(0)=0, \pi \in L^{1}\left(0, T ; H_{1}\right) .
\end{array}\right.
$$

Denote by $\mathcal{R}$ the reachable set of (2.20) at time $T$. Clearly, $\mathcal{R}$ is convex.

Theorem 2.7. Under all the assumptions of Theorem 2.5 suppose that

$$
\operatorname{cl} h_{x}(\bar{x}(T))(\mathcal{R})=H_{2} .
$$

If there exists $w_{T} \in \mathrm{cl} R^{L(2)}$ such that

$$
h_{x}(\bar{x}(T))\left(w_{T}\right)+\frac{1}{2} h_{x x}(\bar{x}(T))(y(T), y(T))=0
$$


and

$$
g_{j, x}(\bar{x}(T))\left(w_{T}\right)+\frac{1}{2} g_{j, x x}(\bar{x}(T))(y(T), y(T))<0, \quad \text { for } j \in \mathcal{I}_{g},
$$

then the conclusion of Theorem 2.5 is valid with $\alpha_{0}=1$.

If there is no $w_{T}$ as in Theorem 2.7, then the second-order condition holds true in the abnormal form. More generally, we have the following result.

Theorem 2.8. Assume (H1), (H3) and let $(\bar{x}, \bar{u})$ be a trajectory-control pair of (1.1) satisfying (2.18). Let $(y, u) \in \Xi$ be a trajectory-control pair of $(2.6)$ such that $\left(\boldsymbol{H}_{4}\right)$ holds and $V^{2}(\bar{u}, u) \cap L^{2}\left(0, T ; H_{1}\right) \neq \emptyset$. If there is no $w_{T}$ as in Theorem 2.7, then the conclusion of Theorem 2.5 is valid with $\alpha_{0}=0$.

For the sake of completeness we also provide a result without assumption (2.17).

Theorem 2.9. Assume (H1), (H3) and let $(\bar{x}, \bar{u})$ be a local minimizer of (1.5) satisfying (2.18). If $\operatorname{cl} h_{x}(\bar{x}(T))\left(R^{L}\right)$ has interior points or is contained in a closed subspace $\widetilde{H}_{2} \varsubsetneqq H_{2}$, then for every $(y, u) \in$ $\mathcal{C}(\bar{x}, \bar{u})$ verifying (H4) and $V^{2}(\bar{u}, u) \cap L^{2}\left(0, T ; H_{1}\right) \neq \emptyset$, there exists $(\alpha, \beta, p) \in \Lambda(\bar{x}, \bar{u})$ such that for any trajectory-control pair $(w, v)$ of (2.13) satisfying $(2.22)$ with $w_{T}=w(T)$, we have

$$
\frac{1}{2} \Omega(x, u, \alpha, \beta, p)+\int_{0}^{T} \mathcal{H}_{u}[t](v(t)) \mathrm{d} t \geq 0 .
$$

In Theorems 2.5-2.9, we assume that $f, g_{j}(j=0, \ldots, r)$ and $h$ are twice continuously Fréchet differentiable with respect to $x$. This is quite restrictive in the infinite dimensional framework, but is needed when $\left\{e^{A t}\right\}_{t \geq 0}$ is a general $C_{0}$-semigroup. On the other hand, if $\left\{e^{A t}\right\}_{t \geq 0}$ has some smoothing effect, then (H3) can be relaxed. Let us discuss it below.

Let $X \subset H$ be a separable reflexive Banach space with the norm Gateaux differentiable away from zero. Further suppose that $X$ is dense in $H$ and that the embedding from $X$ to $H$ is continuous.

Several assumptions are in order.

(S1) $\left\{e^{A t}\right\}_{t \geq 0}$ is a $C_{0}$-semigroup on $X$ and for every $t>0$, there is a constant $C=C(t)>0$ such that for all $\eta \in H,\left|e^{A t} \eta\right|_{X} \leq C|\eta|_{H}$. Further, there exists a constant $\widetilde{C}>0$ such that for all $t \in[0, T)$ and $\eta \in H$, $\left|e^{A \cdot} \eta\right|_{L^{2}(t, T ; X)} \leq \widetilde{C}|\eta|_{H}$.

(S2) $f(\cdot, \cdot, \cdot):[0, T] \times X \times H_{1} \rightarrow X$ is measurable in the first variable, continuous in the third variable, and

$$
\left\{\begin{array}{l}
\left|f\left(t, x_{1}, u\right)-f\left(t, x_{2}, u\right)\right|_{X} \leq C\left|x_{1}-x_{2}\right|_{X}, \quad \forall\left(t, x_{1}, x_{2}, u\right) \in[0, T] \times X \times X \times U \\
|f(t, 0, u)|_{X} \leq C, \quad \forall(t, u) \in[0, T] \times U
\end{array}\right.
$$

(S3) For a.e. $t \in[0, T], f(t, \cdot, \cdot): X \times H_{1} \rightarrow H$ is $C^{2}$. Moreover,

$$
\begin{aligned}
& \left|f_{x}(t, x, u)\right|_{\mathcal{L}(X ; H)}+\left|f_{x x}(t, x, u)\right|_{\mathcal{L}(X, X ; H)}+\left|f_{x u}(t, x, u)\right|_{\mathcal{L}\left(X, H_{1} ; H\right)} \\
& +\left|f_{u}(t, x, u)\right|_{\mathcal{L}\left(H_{1} ; H\right)}+\left|f_{u u}(t, x, u)\right|_{\mathcal{L}\left(H_{1}, H_{1} ; H\right)} \leq C, \quad \forall(t, x, u) \in[0, T] \times X \times U .
\end{aligned}
$$

(S4) $g_{j}: X \rightarrow \mathbb{R}(j=0, \ldots, r)$ and $h: X \rightarrow H_{2}$ are $C^{2}$. Moreover, for all $x \in X$

$$
\sum_{j=0}^{r}\left(\left|g_{j, x}(x)\right|_{\mathcal{L}(X ; \mathbb{R})}+\left|g_{j, x x}(x)\right|_{\mathcal{L}(X, X ; \mathbb{R})}\right)+\left|h_{x}(x)\right|_{\mathcal{L}\left(X ; H_{2}\right)}+\left|h_{x x}(x)\right|_{\mathcal{L}\left(X, X ; H_{2}\right)} \leq C
$$


By (S1)-(S2), it is easy to prove that for any $x_{0} \in H$ and $u \in \mathcal{U}$ there exists a unique mild solution $x(\cdot) \in C([0, T] ; H) \cap L^{2}(0, T ; X)$ to the system $(1.1)$ and $x(t) \in X$ for every $t \in(0, T]$. Furthermore, for any trajectory-control pair $(y, u)$ of $(2.6)$ we have $y \in C([0, T] ; X)$. Similar statement is also valid for trajectories of (2.7). Thus the system in (2.13) is well defined. Moreover $\mathcal{R}, R^{L}, R^{L(2)}$ are subsets of $X$.

By (S4), the final data of the adjoint equation (2.2) belongs to $X^{\prime}$. By (S1) and Corollary 1.10.6 of [16], $A^{*}$ is the infinitesimal generator of the adjoint $C_{0}$-semigroup $\left(e^{A t}\right)^{*}$ on $X^{\prime}$, denoted by $\left\{e^{A^{*}}\right\}_{t \geq 0}$. Also by (S1) for every $t>0$, there is a constant $C=C(t)>0$ such that for all $\eta \in X^{\prime},\left|e^{A^{*} t} \eta\right|_{H} \leq C|\eta|_{X^{\prime}}$ and $e^{A^{*}} \eta \in L^{2}(0, T ; H)$. Further, we have that for all $t \in[0, T)$ and $\eta \in X^{\prime},\left|e^{A^{*}} \eta\right|_{L^{2}(t, T ; H)} \leq \widetilde{C}|\eta|_{X^{\prime}}$. Then it is easy to prove that under assumptions (S1) - (S4) the mild solution to (2.2) belongs to $C\left([0, T] ; X^{\prime}\right) \cap L^{2}(0, T ; H)$ and $p(t) \in H$ for every $t \in[0, T)$.

Define the convex sets

$$
\Theta_{X}=\left\{\eta \in X \mid h_{x}(\bar{x}(T))(\eta)+\frac{1}{2} h_{x x}(\bar{x}(T))(y(T), y(T))=0\right\}
$$

and

$$
\widetilde{\Theta}_{X}=\left\{\theta-\kappa \mid \theta \in \Theta_{X}, \kappa \in \operatorname{cl}_{X}\left(R^{L(2)}\right)\right\},
$$

where $\mathrm{cl}_{X}$ denotes the closure in $X$.

We impose the following assumption.

(S5) There is a closed subspace $\widetilde{X}$ of $X$ such that $\widetilde{\Theta}_{X} \subset \widetilde{X}$ and $\operatorname{int}_{\widetilde{X}} \widetilde{\Theta}_{X} \neq \emptyset$, where $\operatorname{int}_{\tilde{X}} \widetilde{\Theta}_{X}$ denotes the interior of $\widetilde{\Theta}_{X}$ in $\widetilde{X}$.

Corresponding to Theorems 2.5-2.9, we have the following results with

$$
\begin{gathered}
K_{j} \triangleq\left\{x \in X \mid g_{j}(x) \leq 0\right\} \text { for } j=1, \ldots, r, \\
\mathcal{I}_{g} \triangleq\left\{j=1, \ldots, r \mid \bar{x}(T) \in \partial_{X} K_{j}\right\},
\end{gathered}
$$

where $\partial_{X} K_{j}$ denotes the boundary of $K_{j}$ in $X$ and

$$
\Lambda_{X}(\bar{x}, \bar{u}) \triangleq\left\{(\alpha, \beta, p) \in \mathbb{R}_{+}^{r+1} \times H_{2} \times C\left([0, T] ; X^{\prime}\right) \mid(\alpha, \beta, p) \neq 0 \text { and satisfies }(2.1)-(2.3)\right\} .
$$

Theorem 2.10. Suppose (S1)-(S4) and let $(\bar{x}, \bar{u})$ be a local minimizer of (1.5) satisfying (2.17) and

$$
h_{x}(\bar{x}(T))(X)=H_{2} .
$$

Let $(y, u) \in \mathcal{C}(\bar{x}, \bar{u})$ be such that (S5) holds and $V^{2}(\bar{u}, u) \cap L^{2}\left(0, T ; H_{1}\right) \neq \emptyset$. Then there exists $(\alpha, \beta, p) \in$ $\Lambda_{X}(\bar{x}, \bar{u})$ such that $\alpha_{j}=0$ whenever $g_{j, x}(\bar{x}(T))(y(T))<0$, the function $\Upsilon(u, p)$ is integrable and

$$
\frac{1}{2} \Omega(y, u, \alpha, \beta, p)+\int_{0}^{T} \Upsilon(u(t), p(t)) \mathrm{d} t \geq 0 .
$$

Theorem 2.11. Suppose (S1)-(S4) and let $(\bar{x}, \bar{u})$ be a local minimizer of (1.5) satisfying (2.17), (2.21). Let $(y, u) \in \mathcal{C}(\bar{x}, \bar{u})$ be such that (S5) is satisfied and $V^{2}(\bar{u}, u) \cap L^{2}\left(0, T ; H_{1}\right) \neq \emptyset$. If there exists $w_{T} \in \operatorname{cl}_{X} R^{L(2)}$ such that (2.22) and (2.23) hold, then the conclusion of Theorem 2.10 is valid with $\alpha_{0}=1$. 
Theorem 2.12. Suppose (S1)-(S4). Let $(\bar{x}, \bar{u})$ be a trajectory-control pair of (1.1) satisfying (2.30) and $(y, u) \in \Xi$ be a trajectory-control pair of (2.6) such that (S5) holds and $V^{2}(\bar{u}, u) \cap L^{2}\left(0, T ; H_{1}\right) \neq \emptyset$. If there is no $w_{T}$ as in Theorem 2.11, then the conclusion of Theorem 2.10 is valid with $\alpha_{0}=0$.

Theorem 2.13. Suppose (S1)-(S4) and let $(\bar{x}, \bar{u})$ be a local minimizer of (1.5) satisfying (2.30). If $\operatorname{cl} h_{x}(\bar{x}(T))\left(R^{L}\right)$ has interior points or is contained in a closed subspace $\widetilde{H}_{2} \varsubsetneqq H_{2}$, then for every $(y, u) \in$ $\mathcal{C}(\bar{x}, \bar{u})$ satisfying (S5) and $V^{2}(\bar{u}, u) \cap L^{2}\left(0, T ; H_{1}\right) \neq \emptyset$, there exists $(\alpha, \beta, p) \in \Lambda_{X}(\bar{x}, \bar{u})$ such that for any trajectory-control pair $(w, v)$ of (2.13) verifying $(2.22)$ with $w_{T}=w(T)$, we have

$$
\frac{1}{2} \Omega(y, u, \alpha, \beta, p)+\int_{0}^{T} \mathcal{H}_{u}[t](v(t)) \mathrm{d} t \geq 0
$$

Proofs of Theorems 2.10-2.13 are very similar to those of Theorems 2.5-2.9, respectively. To shorten the paper, we only give the one of Theorem 2.10 .

\section{Preliminaries}

\subsection{Some results of set-valued analysis}

For readers' convenience, we collect some basic facts from set-valued analysis. More information can be found in [1].

Definition 3.1. Let $(\mathcal{M}, \Sigma)$ be a measurable space, $Z$ a separable Banach space and $F: \mathcal{M} \rightsquigarrow Z$ a set-valued map. For any $\omega \in \mathcal{M}, F(\omega)$ is called the value of $F$ at $\omega$. The map $F$ is called measurable if

$$
F^{-1}(B) \triangleq\{\omega \in \mathcal{M} \mid F(\omega) \cap B \neq \emptyset\} \in \Sigma \text { for any } B \in \mathcal{B}(Z)
$$

where $\mathcal{B}(Z)$ is the Borel $\sigma$-algebra on $Z$.

Definition 3.2. Let $(\mathcal{M}, \Sigma, \mu)$ be a complete $\sigma$-finite measure space, $Y$ and $Z$ two complete separable metric spaces. We call $g: \mathcal{M} \times Y \rightarrow Z$ a Carathéodory function if for every $\omega \in \mathcal{M}, g(\omega, \cdot)$ is continuous and for every $y \in Y, g(\cdot, y)$ is measurable.

Lemma 3.3. ([1], Thm. 8.2.8) Let $(\mathcal{M}, \Sigma, \mu)$ be a complete $\sigma$-finite measure space, $Y$ and $Z$ two complete separable metric spaces, and $S: \mathcal{M} \rightsquigarrow Y$ a measurable set-valued map with closed values. Let $g: \mathcal{M} \times Y \rightarrow Z$ be a Carathéodory function. Then the set-valued map defined by

$$
\mathcal{M} \ni \omega \rightsquigarrow \operatorname{cl} g(\omega, S(\omega))
$$

is measurable.

Next, we recall the notion of measurable selection for a set-valued map.

Definition 3.4. Let $(\mathcal{M}, \Sigma)$ be a measurable space and $Z$ a complete separable metric space. Let $F$ be a setvalued map from $\mathcal{M}$ to $Z$. A measurable map $f: \mathcal{M} \rightarrow Z$ is called a measurable selection of $F$ if $f(\omega) \in F(\omega)$ for all $\omega \in \mathcal{M}$.

A sufficient condition for the existence of a measurable selection is as follows.

Lemma 3.5. ([1], Thm. 8.1.3) Let $Z$ be a complete separable metric space, $(\mathcal{M}, \Sigma)$ a measurable space, and $F: \mathcal{M} \rightsquigarrow Z$ a measurable set-valued map with nonempty closed values. Then there exists a measurable selection of $F$. 
Lemma 3.6. ([1], Thm. 8.2.9) Consider a complete $\sigma$-finite measure space $(\mathcal{M}, \Sigma, \mu)$, complete separable metric spaces $Y, Z$, and measurable set-valued maps $S: \mathcal{M} \rightsquigarrow Y, G: \mathcal{M} \rightsquigarrow Z$ with closed values. Let $g: \mathcal{M} \times Y \rightarrow Z$ be a Carathéodory function. Then the set-valued map $R$ defined by

$$
R(\omega) \triangleq\{x \in S(\omega) \mid g(\omega, x) \in G(\omega)\}
$$

is measurable. Consequently, if $R(\omega) \neq \emptyset$ for all $\omega \in \mathcal{M}$, then there exists a measurable selection $f(\omega) \in S(\omega)$ such that $g(\omega, f(\omega)) \in G(\omega)$ for all $\omega \in \mathcal{M}$.

Recall that for a measure space $(\mathcal{M}, \Sigma, \mu)$, the integral of a set-valued map $\omega \rightsquigarrow \Psi(\omega)$ from $\mathcal{M}$ into a Banach space $Z$ is defined by

$$
\int_{\mathcal{M}} \Psi \mathrm{d} \mu=\left\{\int_{\mathcal{M}} \psi(\omega) \mathrm{d} \omega \mid \psi(\omega) \in \Psi(\omega) \text { a.e., } \psi(\cdot) \text { is Bochner integrable }\right\},
$$

where $\int_{\mathcal{M}} \psi(\omega) \mathrm{d} \omega$ is the Bochner integral of $\psi(\cdot)$ over $\mathcal{M}$. We say that $\Psi$ is integrably bounded, if there is a (Lebesgue) integrable $k: \mathcal{M} \rightarrow \mathbf{R}_{+}$such that $\Psi(\omega) \in k(\omega) B_{Z}(1)$ for all $\omega \in \mathcal{M}$, where $B_{Z}(1)$ stands for the closed unit ball in $Z$.

Lemma 3.7. Let $(\mathcal{M}, \Sigma, \mu)$ be a complete measure space with $\mu(\mathcal{M})<\infty, Y$ be a separable metric space, and $Z$ be a separable Banach space. Consider a measurable set-valued map $S: \mathcal{M} \rightsquigarrow Y$ with closed values and a Carathéodory function $g: \mathcal{M} \times Y \rightarrow Z$. If $\mathcal{M} \ni \omega \rightsquigarrow g(\omega, Y)$ is integrably bounded, then

$$
\operatorname{cl} \int_{\mathcal{M}} g(\omega, S(\omega)) \mathrm{d} \mu=\operatorname{cl} \int_{\mathcal{M}} \operatorname{cl} g(\omega, S(\omega)) \mathrm{d} \mu
$$

Proof. Clearly

$$
\operatorname{cl} \int_{\mathcal{M}} g(\omega, S(\omega)) \mathrm{d} \mu \subset \operatorname{cl} \int_{\mathcal{M}} \operatorname{cl} g(\omega, S(\omega)) \mathrm{d} \mu
$$

Fix a Bochner integrable selection $\psi(\omega) \in \operatorname{cl} g(\omega, S(\omega))$ and let $\delta>0$. Then for

$$
B_{Z}(\psi(\omega), \delta) \triangleq\left\{\eta \in Z|| \eta-\left.\psi(\omega)\right|_{Z} \leq \delta\right\}
$$

we have

$$
g(\omega, S(\omega)) \cap B_{Z}(\psi(\omega), \delta) \neq \emptyset \quad \forall \omega \in \mathcal{M} .
$$

By Lemma 3.6, there exists a measurable selection $u(\omega) \in S(\omega)$ such that $g(\omega, u(\omega)) \in B_{Z}(\psi(\omega), \delta)$. Since $u(\cdot)$ is measurable, it is the pointwise limit of simple measurable maps $\left\{u_{n}\right\}_{n \geq 1}$. Using that $g$ is Carathéodory we deduce that $g(\cdot, u(\cdot))$ is pointwise limit of simple measurable maps. Since $g(\omega, Y)$ is integrably bounded, by the Lebesgue dominated convergence theorem, $g(\cdot, u(\cdot))$ is Bochner integrable. This implies that

$$
\int_{\mathcal{M}} \psi \mathrm{d} \mu \in \int_{\mathcal{M}} g(\omega, u(\omega)) \mathrm{d} \mu+\delta \mu(\mathcal{M}) B_{Z}(1)
$$

By arbitrariness of $\delta>0$, we have $\int_{\mathcal{M}} \psi \mathrm{d} \mu \in \mathrm{cl} \int_{\mathcal{M}} g(\omega, S(\omega)) \mathrm{d} \mu$. The arbitrariness of the Bochner integrable selection $\psi(\omega) \in \operatorname{cl} g(\omega, S(\omega))$ ends the proof. 
Lemma 3.8. ([1], Props. 8.6.2 and 8.6.4) Let $(\mathcal{M}, \Sigma, \mu)$ be a complete $\sigma$-finite measure space, $Y$ a separable Banach space, and $F: \mathcal{M} \rightsquigarrow Y$ a measurable, integrably bounded set-valued map with nonempty closed values. Then

$$
\mathrm{cl} \int_{\mathcal{M}} \overline{\mathrm{Co}} F \mathrm{~d} \mu=\overline{\mathrm{co}} \int_{\mathcal{M}} F \mathrm{~d} \mu
$$

Furthermore, if $\mu$ is non-atomic, then

$$
\mathrm{cl} \int_{\mathcal{M}} \overline{\operatorname{co}} F \mathrm{~d} \mu=\mathrm{cl} \int_{\mathcal{M}} F \mathrm{~d} \mu
$$

Corollary 3.9. Suppose that all the assumptions of Lemma 3.7 hold and $\mu$ is non-atomic. Then

$$
\operatorname{cl} \int_{\mathcal{M}} g(\omega, S(\omega)) \mathrm{d} \mu=\mathrm{cl} \int_{\mathcal{M}} \overline{\operatorname{co}} g(\omega, S(\omega)) \mathrm{d} \mu .
$$

As an immediate corollary of Lemma 2.10 of [12], we have the following result.

Lemma 3.10. Let $\Sigma$ be the $\sigma$-algebra of all Lebesgue measurable sets contained in $[0, T], U \subset H_{1}$ be closed and $u \in \mathcal{U}$. Then the set-valued map $t \rightsquigarrow \mathcal{C}_{U}(u(t))$ is $\Sigma$ measurable.

We shall also need the following lemma.

Lemma 3.11. ([9], Lem. 2.4) Let $Z$ be a Banach space, $\mathcal{K} \subset Z$. Then for every $z \in \mathcal{K} \subset Z$ and $z_{1} \in T_{\mathcal{K}}^{b}(z)$,

$$
T_{\mathcal{K}}^{b(2)}\left(z, z_{1}\right)=T_{\mathcal{K}}^{b(2)}\left(z, z_{1}\right)+\mathcal{C}_{\mathcal{K}}(z)
$$

\subsection{Some inverse mapping theorems}

The aim of this section is to prove the following two inverse mapping like theorems.

Theorem 3.12. Assume (H1), (H3) and let $(\bar{x}, \bar{u})$ be a trajectory-control pair of $(1.1)$ with $h(\bar{x}(T))=0$. If (2.17) holds true, then there exist $\varepsilon>0$ and $c>0$ such that for every trajectory-control pair $(x, u)$ of $(1.1)$ satisfying $|u-\bar{u}|_{L^{1}\left(0, T: H_{1}\right)}<\varepsilon$ we can find a trajectory-control pair $(\tilde{x}, \tilde{u})$ of (1.1) satisfying

$$
h(\tilde{x}(T))=0, \quad|\tilde{u}-u|_{L^{1}\left(0, T ; H_{1}\right)} \leq c|h(x(T))|_{H_{2}} .
$$

Theorem 3.13. Assume (S1)-(S4) and let $(\bar{x}, \bar{u})$ be a trajectory-control pair of $(1.1)$ with $h(\bar{x}(T))=0$. If (2.17) holds true, then there exist $\varepsilon>0$ and $C>0$ such that for every trajectory-control pair $(x, u)$ of $(1.1)$ with $|u-\bar{u}|_{L^{1}\left(0, T: H_{1}\right)}<\varepsilon$ we can find a trajectory-control pair $(\tilde{x}, \tilde{u})$ of (1.1) satisfying

$$
h(\tilde{x}(T))=0, \quad|\tilde{u}-u|_{L^{1}\left(0, T ; H_{1}\right)} \leq C|h(x(T))|_{H_{2}} .
$$

By the classical theory of evolution equations (e.g. [13], Chap. 2, Subsect. 5.2), we know that under assumptions (H1) and (H2), to every control $u \in \mathcal{U}$ there corresponds a unique solution $x(\cdot, u)$ of the control system (1.1). Furthermore, using the Gronwall lemma, it is not difficult to check that the mapping $\mathcal{U} \ni u \mapsto x(\cdot ; u) \in C([0, T] ; H)$ is continuous. However, since $\mathcal{U}$ is only a metric space, one cannot differentiate the map $\mathcal{U} \ni u \rightarrow h(x(T, u))$ to get the above inverse mapping theorems. Instead we replace derivatives by variations in the following way: 
Recall that for a family of subsets $\left\{\Gamma_{\delta}\right\}_{\delta>0}$ of a Banach space $Z$, the upper set limit is defined by

$$
v \in \operatorname{Limsup}_{\delta \rightarrow 0^{+}} \Gamma_{\delta} \Longleftrightarrow \liminf _{\delta \rightarrow 0^{+}} \operatorname{dist}\left(v, \Gamma_{\delta}\right)=0 .
$$

Define the continuous map $G: \mathcal{U} \rightarrow H_{2}$ by $G(u) \triangleq h(x(T, u))$. For $\delta>0$ and $u \in \mathcal{U}$, set

$$
B_{\delta}(u) \triangleq\{v \in \mathcal{U} \mid \mathbf{d}(v, u) \leq \delta\} .
$$

The first-order contingent variation of $G$ at $u \in \mathcal{U}$ is defined by

$$
G^{(1)}(u) \triangleq \operatorname{Limsup}_{\delta \rightarrow 0^{+}} \frac{G\left(B_{\delta}(u)\right)-G(u)}{\delta} .
$$

Usually, it is difficult to compute the whole set $G^{(1)}(u)$. However, as we show below, the set $h_{x}(\bar{x}(T))\left(R^{L}\right)$ is a subset of $\overline{c o} G^{(1)}(\bar{u})$. This is sufficient for our purposes.

Denote by $B^{H_{2}}$ the closed unit ball in $H_{2}$. The following result is an immediate consequence of Theorem 3.2 of $[7]$.

Lemma 3.14. If for some $\varepsilon>0$ and $\rho>0, \rho B^{H_{2}} \subset \bigcap_{\mathbf{d}(u, \bar{u}) \leq \varepsilon} \overline{\operatorname{co}} G^{(1)}(u)$, then for every $u \in \mathcal{U}$ such that $\mathbf{d}(\bar{u}, u) \leq$ $\frac{\varepsilon}{2}$ and $c \in\left[0, \frac{\varepsilon}{2}\right]$,

$$
G(u)+c \rho \operatorname{int} B^{H_{2}} \subset G\left(B_{c}(u)\right) .
$$

Moreover, for every $u \in B_{\frac{\varepsilon}{4}}(\bar{u})$ and $x \in H_{2}$ satisfying $|x-G(u)|_{H_{2}}<\min \left\{\frac{\varepsilon}{8}, \frac{\varepsilon \rho}{4}\right\}$, we have

$$
\operatorname{dist}\left(u, G^{-1}(x)\right) \leq \frac{1}{\rho}|x-G(u)|_{H_{2}} .
$$

Lemma 3.15. Assume (H1), (H3) and let $(\bar{x}, \bar{u})$ be a trajectory-control pair of (1.1). If (2.17) holds true, then there exist $\varepsilon>0$ and $\rho>0$ such that for every $u \in \mathcal{U}$ satisfying $\mathbf{d}(u, \bar{u})<\varepsilon$, we have $\rho B^{H_{2}} \subset \overline{\mathrm{co}} G^{(1)}(u)$.

Before proving Lemma 3.15, we recall the following result.

Lemma 3.16. ([2], Thm. 2.8) Let $Y$ and $Z$ be separable metric spaces and consider a Carathéodory map $F:[0, T] \times Y \rightarrow Z$. Then, for any $\varepsilon>0$ there exists a compact set $\mathcal{T}_{\varepsilon} \subset[0, T]$ with the Lebesgue measure of $\left([0, T] \backslash \mathcal{T}_{\varepsilon}\right)$ smaller than $\varepsilon$ and such that the restriction of $F$ to $\mathcal{T}_{\varepsilon} \times Y$ is continuous.

Proof of Lemma 3.15. For $u \in \mathcal{U}$, let $x(\cdot, u)$ denote the corresponding solution of (1.1). By (H1) the set $\{x(\cdot, u)\}_{u \in \mathcal{U}}$ is bounded in $C([0, T] ; H)$. We borrow some ideas from the proof of Proposition 2.9 in [2]. Choosing $Y=H \times H_{1}, Z=H$ and $F=f$, by Lemma 3.16, for every $k \in \mathbb{N}$, there exists a compact $\mathcal{T}_{1, k} \subset[0, T]$ such that $f$ is continuous on $\mathcal{T}_{1, k} \times H \times H_{1}$ and the Lebesgue measure of $[0, T] \backslash \mathcal{T}_{1, k}$ is smaller than $\frac{1}{k}$. Fix any $u \in \mathcal{U}$. By Lemma 3.16 again, for every $k \in \mathbb{N}$, there exists a compact $\mathcal{T}_{2, k} \subset[0, T]$ such that $u$ is continuous on $\mathcal{T}_{2, k}$ and the Lebesgue measure of $[0, T] \backslash \mathcal{T}_{2, k}$ is smaller than $\frac{1}{k}$. Let $\mathcal{T}_{k}=\mathcal{T}_{1, k} \cap \mathcal{T}_{2, k}$ and denote by $\widetilde{\mathcal{T}}_{k}$ the set of Lebesgue density points in $\mathcal{T}_{k}$. Then the Lebesgue measure of the set $\mathcal{T} \triangleq \bigcup_{k=1}^{\infty} \widetilde{\mathcal{T}}_{k}$ is equal to $T$.

Define $M=\max _{w \in U}|w|_{H_{1}}+1$ and consider a dense subset $\left\{u_{j}\right\}_{j=1}^{\infty}$ of $U$. Let $t \in \mathcal{T} \cap(0, T]$ and $\delta \in(0,2 M t)$. For a fixed $j \in \mathbb{N}$, define the control

$$
u_{j, \delta}(s)= \begin{cases}u_{j}, & \text { if } s \in[t-\delta / 2 M, t] \\ u(s), & \text { otherwise. }\end{cases}
$$


Then $u_{j, \delta} \in B_{\delta}(u)$ and

$$
\begin{aligned}
x\left(t, u_{j, \delta}\right)-x(t, u) & =\int_{t-\delta / 2 M}^{t} e^{A(t-s)}\left[f\left(s, x\left(s, u_{j}\right), u_{j}\right)-f(s, x(s, u), u(s))\right] \mathrm{d} s \\
& =\int_{t-\delta / 2 M}^{t} e^{A(t-s)}\left[f\left(s, x\left(s, u_{j}\right), u_{j}\right)-f\left(s, x(s, u), u_{j}\right)\right] \mathrm{d} s \\
& +\int_{t-\delta / 2 M}^{t} e^{A(t-s)}\left[f\left(s, x(s, u), u_{j}\right)-f(s, x(s, u), u(s))\right] \mathrm{d} s .
\end{aligned}
$$

From (H3), we deduce that

$$
\begin{aligned}
& \left|\int_{t-\delta / 2 M}^{t} e^{A(t-s)}\left[f\left(s, x\left(s, u_{j}\right), u_{j}\right)-f\left(s, x(s, u), u_{j}\right)\right] \mathrm{d} s\right|_{H} \\
& =\left|\int_{t-\delta / 2 M}^{t} e^{A(t-s)} \int_{0}^{1} f_{x}\left(s, x(s, u)+\sigma\left(x\left(s, u_{j}\right)-x(s, u)\right), u_{j}\right)\left(x\left(s, u_{j}\right)-x(s, u)\right) \mathrm{d} \sigma \mathrm{d} s\right|_{H} \\
& \leq C \sup _{s \in[t-\delta / 2 M, t]}\left|x\left(s, u_{j}\right)-x(s, u)\right|_{H} \delta=o(\delta) .
\end{aligned}
$$

Since $t \in \mathcal{T}$, there exists $k>0$ such that $t \in \widetilde{\mathcal{T}}_{k}$. Since $t$ is a Lebesgue density point of $\mathcal{T}_{k}$, it follows from (H1) that

$$
\begin{aligned}
& \lim _{\delta \rightarrow 0}\left|\frac{1}{\delta} \int_{[t-\delta / 2 M, t] \backslash \mathcal{T}_{k}} e^{A(t-s)}\left[f\left(s, x(s, u), u_{j}\right)-f(s, x(s, u), u(s))\right] \mathrm{d} s\right|_{H} \\
& \leq C \lim _{\delta \rightarrow 0} \frac{1}{\delta} \int_{[t-\delta / 2 M, t] \backslash \mathcal{T}_{k}} \mathrm{~d} s=0 .
\end{aligned}
$$

Recalling that $f(\cdot, \cdot, \cdot)$ is continuous on $\mathcal{T}_{k} \times H \times H_{1}$, from (3.3), we obtain

$$
\begin{aligned}
& \lim _{\delta \rightarrow 0} \frac{1}{\delta} \int_{t-\delta / 2 M}^{t} e^{A(t-s)}\left[f\left(s, x(s, u), u_{j}\right)-f(s, x(s, u), u(s))\right] \mathrm{d} s \\
& =\lim _{\delta \rightarrow 0} \frac{1}{\delta} \int_{[t-\delta / 2 M, t] \cap \mathcal{T}_{k}} e^{A(t-s)}\left[f\left(s, x(s, u), u_{j}\right)-f(s, x(s, u), u(s))\right] \mathrm{d} s \\
& =\frac{1}{2 M}\left[f\left(t, x(t, u), u_{j}\right)-f(t, x(t, u), u(t))\right] .
\end{aligned}
$$

Combining (3.1), (3.2) and (3.4), we have

$$
\lim _{\delta \rightarrow 0} \frac{x\left(t, u_{j, \delta}\right)-x(t, u)}{\delta}=\frac{1}{2 M}\left[f\left(t, x(t, u), u_{j}\right)-f(t, x(t, u), u(t))\right] .
$$

Consider the following equation:

$$
\left\{\begin{array}{l}
z_{t}(s)=A z(s)+f_{x}(s, x(s, u), u(s)) z(s), \quad s \in(t, T], \\
z(t)=z_{0}
\end{array}\right.
$$


and define the family of operators $\Phi(\cdot, t ; u)$ on $H$ as follows:

$$
\Phi(s, t ; u) z_{0}=z(s) \quad \text { for } s \in[t, T] .
$$

Then we have that

$$
\lim _{\delta \rightarrow 0^{+}} \frac{x\left(T, u_{j, \delta}\right)-x(T, u)}{\delta}=\frac{1}{2 M} \Phi(T, t ; u)\left[f\left(t, x(t, u), u_{j}\right)-f(t, x(t, u), u(t))\right]
$$

Therefore,

$$
h_{x}(x(T, u))\left\{\Phi(T, t ; u)\left[f\left(t, x(t, u), u_{j}\right)-f(t, x(t, u), u(t))\right]\right\} \subset 2 M G^{(1)}(u) .
$$

Since $j \in \mathbb{N}$ is arbitrary, $\left\{u_{j}\right\}_{j=1}^{\infty}$ is dense in $U$, and $f$ is continuous with respect to the third variable, it follows from (3.7) that for a.e. $t \in[0, T]$,

$$
h_{x}(x(T, u))\{\Phi(T, t ; u)[f(t, x(t, u), U)-f(t, x(t, u), u(t))]\} \subset 2 M \overline{\mathrm{co}} G^{(1)}(u) .
$$

Consequently, for every measurable selection $v(t) \in f(t, x(t, u), U)-f(t, x(t, u), u(t))$ and for a.e. $t \in[0, T]$, we have

$$
h_{x}(x(T, u))[\Phi(T, t ; u) v(t)] \in 2 M \overline{\mathrm{co}} G^{(1)}(u)
$$

This implies that

$$
h_{x}(x(T, u))\left[\int_{0}^{T} \Phi(T, t ; u) v(t) \mathrm{d} t\right] \in 2 M T \overline{\mathrm{co}} G^{(1)}(u) .
$$

Denote by $R^{L, u}$ the reachable set at time $T$ of the following control system:

$$
\left\{\begin{array}{l}
\hat{y}_{t}(t)=A \hat{y}(t)+f_{x}(t, x(t, u), u(t)) \hat{y}(t)+v(t), \quad t \in(0, T] \\
v(t) \in f(x(t, u), U)-f(x(t, u), u(t)), \quad \text { a.e. } t \in[0, T] \\
\hat{y}(0)=0
\end{array}\right.
$$

Since $\overline{\mathrm{co}} G^{(1)}(u)$ is closed, we get from (3.8) that

$$
\operatorname{cl} h_{x}(x(T, u))\left(R^{L, u}\right) \subset 2 M T \overline{\operatorname{co}} G^{(1)}(u) .
$$

Hence, we only need to show that

$$
\exists \bar{\rho}>0, \forall u \in \mathcal{U} \text {, sufficiently close to } \bar{u} \text { in } \mathcal{U} \text {, it holds that } \bar{\rho} B^{H_{2}} \subset \operatorname{cl} h_{x}(x(T, u))\left(R^{L, u}\right) .
$$

By the continuity of the map $\mathcal{U} \ni u \rightarrow x(\cdot, u) \in C([0, T] ; H)$ we know that

$$
\forall \hat{\varepsilon}>0, \exists \hat{\delta}>0 \text { such that }|x(\cdot, u)-\bar{x}(\cdot)|_{C([0, T] ; H)}<\hat{\varepsilon}, \forall u \in \mathcal{U} \text { with } \mathbf{d}(u, \bar{u})<\hat{\delta} .
$$


Using the Gronwall lemma it is not difficult to show that

$$
\forall \hat{\varepsilon}>0, \exists \hat{\delta}>0 \text { such that } \sup _{t \in[0, T]}|\Phi(T, t ; u)-\Phi(T, t ; \bar{u})|_{\mathcal{L}(H)}<\hat{\varepsilon}, \forall u \in \mathcal{U} \text { with } \mathbf{d}(u, \bar{u})<\hat{\delta} .
$$

Let $\tilde{u} \in \mathcal{U}$. From (3.11), (3.12) and (H3) we deduce that for each $\varepsilon>0$, there exists $\delta=\delta(\varepsilon)>0$ independent from $\tilde{u}$ such that for any $u \in \mathcal{U}$ satisfying $\mathbf{d}(u, \bar{u})<\delta$,

$$
\begin{aligned}
& h_{x}(\bar{x}(T))\left\{\int_{0}^{T} \Phi(T, t ; \bar{u})[f(t, \bar{x}(t), \tilde{u}(t))-f(t, \bar{x}(t), \bar{u}(t))] \mathrm{d} t\right\} \\
& \in h_{x}(x(T, u))\left\{\int_{0}^{T} \Phi(T, t ; u)[f(t, x(t, u), \tilde{u}(t))-f(t, x(t, u), u(t))] \mathrm{d} t\right\}+\varepsilon B^{H_{2}} .
\end{aligned}
$$

Observe next that by Lemmas 3.5 and 3.6 for every measurable selection $v(t) \in f(t, \bar{x}(t), U)-f[t]$ there exists $\tilde{u} \in \mathcal{U}$ such that $v(t)=f(t, \bar{x}(t), \tilde{u}(t))-f[t]$ a.e. in $[0, T]$. Consequently,

$$
\operatorname{cl} h_{x}(\bar{x}(T))\left(R^{L, \bar{u}}\right) \subset \operatorname{cl}\left(h_{x}(x(T, u))\left(R^{L, u}\right)+\varepsilon B^{H_{2}}\right) .
$$

Define $F(t)=f(t, \bar{x}(t), U)-f[t]$ for all $t \in[0, T]$. It is not difficult to check that

$$
\overline{\mathrm{co}}\left(h_{x}(\bar{x}(T)) \Phi(T, t ; u) F(t)\right)=\operatorname{cl}\left(h_{x}(\bar{x}(T)) \Phi(T, t ; u) \overline{\operatorname{co}} F(t)\right) .
$$

Therefore, by Lemma 3.7, applied with $S(t)=\overline{\operatorname{co}} F(t)$ and $g(t, \xi)=h_{x}(\bar{x}(T)) \Phi(T, t ; \bar{u}) \xi$

$$
\begin{aligned}
\operatorname{cl}\left(h_{x}(\bar{x}(T)) R^{L}\right) & =\operatorname{cl} \int_{0}^{T} h_{x}(\bar{x}(T)) \Phi(T, t ; \bar{u}) \overline{\operatorname{co}} F(t) \mathrm{d} t \\
& =\operatorname{cl} \int_{0}^{T} \operatorname{cl}\left(h_{x}(\bar{x}(T)) \Phi(T, t ; \bar{u}) \overline{\operatorname{co}} F(t)\right) \mathrm{d} t \\
& =\operatorname{cl} \int_{0}^{T} \overline{\operatorname{co}}\left(h_{x}(\bar{x}(T)) \Phi(T, t ; \bar{u}) F(t)\right) \mathrm{d} t .
\end{aligned}
$$

This and Corollary 3.9 applied with $S \equiv U$ and $g(t, u)=h_{x}(\bar{x}(T)) \Phi(T, t ; \bar{u})(f(t, \bar{x}(t), u)-f[t])$ imply that

$$
\operatorname{cl}\left(h_{x}(\bar{x}(T)) R^{L}\right)=\operatorname{cl} \int_{0}^{T} h_{x}(\bar{x}(T)) \Phi(T, t ; \bar{u}) F(t) \mathrm{d} t
$$

Therefore $\mathrm{cl}\left(h_{x}(\bar{x}(T)) R^{L}\right)=\mathrm{cl}\left(h_{x}(\bar{x}(T)) R^{L, \bar{u}}\right)$ and the set $\mathrm{cl}\left(h_{x}(\bar{x}(T)) R^{L, \bar{u}}\right)$ is convex. The same argument implies that $\mathrm{cl}\left(h_{x}(\bar{x}(T)) R^{L, u}\right)$ is convex for any $u \in \mathcal{U}$.

By (2.17), for some $\rho>0$ we have

$$
\rho B^{H_{2}} \subset \operatorname{cl}\left(h_{x}(\bar{x}(T)) R^{L, \bar{u}}\right) .
$$

Fix $\varepsilon \in\left(0, \frac{\rho}{2}\right)$ and let $\delta=\delta(\varepsilon)$ be as above.

We claim that

$$
(\rho-2 \varepsilon) B^{H_{2}} \subset \operatorname{cl} h_{x}(x(T, u))\left(R^{L, u}\right), \quad \forall u \in \mathcal{U} \text { satisfying } \mathbf{d}(u, \bar{u})<\delta .
$$


Otherwise, by the separation theorem, there exists $q_{1} \in H_{2}$ with $\left|q_{1}\right|_{H_{2}}=1$ such that

$$
\sup _{e \in h_{x}(x(T ; u))\left(R^{L, u}\right)}\left\langle e, q_{1}\right\rangle_{H_{2}}<\rho-2 \varepsilon .
$$

By (3.13),

$$
\sup _{e \in h_{x}(\bar{x}(T))\left(R^{L, \bar{u}}\right)}\left\langle e, q_{1}\right\rangle_{H_{2}} \leq \sup _{e \in h_{x}(x(T ; u))\left(R^{L, u}\right)}\left\langle e, q_{1}\right\rangle_{H_{2}}+\varepsilon
$$

This, together with (3.14), yields

$$
\rho \leq \sup _{e \in h_{x}(\bar{x}(T))\left(R^{L, \bar{u}}\right)}\left\langle e, q_{1}\right\rangle_{H_{2}} \leq \sup _{e \in h_{x}(x(T ; u))\left(R^{L, u}\right)}\left\langle e, q_{1}\right\rangle_{H_{2}}+\varepsilon<\rho-2 \varepsilon+\varepsilon=\rho-\varepsilon .
$$

The obtained contradiction yields (3.15). Consequently, (3.10) is satisfied with $\bar{\rho}=\rho-2 \varepsilon$ whenever $\mathbf{d}(u, \bar{u})<\delta$.

Proof of Theorem 3.12. By Lemma 3.15, there exist $\varepsilon>0$ and $\rho>0$ such that for every $u \in \mathcal{U}$ with $\mathbf{d}(u, \bar{u})<\varepsilon$ we have $\rho B^{H_{2}} \subset \overline{\mathrm{co}} G^{(1)}(u)$. Then by Lemma 3.14, we obtain the desired result.

To prove Theorem 3.13, we need to modify the assumptions of Lemma 3.15.

Lemma 3.17. Assume $(\boldsymbol{S 1})-\left(\boldsymbol{S}_{4}\right)$ and let $(\bar{x}, \bar{u})$ be a trajectory-control pair of (1.1). If (2.17) holds true, then there exist $\varepsilon>0, \rho>0$ such that for every $u \in \mathcal{U}$ satisfying $\mathbf{d}(u, \bar{u})<\varepsilon$ we have $\rho B^{H_{2}} \subset \overline{\mathrm{co}} G^{(1)}(u)$.

Proof. By (S1)-(S2), for every $u \in \mathcal{U}$ and $t \in(0, T], x(t, u) \in X$. Then one can mimic the proof of Lemma 3.15 to deduce Lemma 3.17 .

The proof of Theorem 3.13 is the same as the one of Theorem 3.12.

\subsection{Tangents to trajectories satisfying end-point equality constraints}

The aim of this section is to prove the following two lemmas.

Lemma 3.18. Assume (H1) and (H3). Let $(\bar{x}, \bar{u})$ be a trajectory-control pair of $(1.1)$ with $h(\bar{x}(T))=0$ satisfying (2.17) and $(y, u) \in \Xi$ be a trajectory-control pair of (2.6) satisfying $(2.11)$ and such that $h_{x}(\bar{x}(T))(y(T))=0$. Then for every trajectory-control pair $(w, v) \in \Xi$ of (2.13) satisfying (2.22) with $w_{T}=w(T)$, there exists $c>0$ and trajectory-control pairs $\left\{\left(x_{\delta}, u_{\delta}\right)\right\}_{\delta>0}$ of (1.1) such that $\left|u_{\delta}-\bar{u}\right|_{L^{1}\left(0, T ; H_{1}\right)} \leq c \delta$,

$$
h\left(x_{\delta}(T)\right)=0
$$

and

$$
\lim _{\delta \rightarrow 0+} \frac{\left|x_{\delta}-\bar{x}-\delta y-\delta^{2} w\right|_{C([0, T] ; H)}}{\delta^{2}}=0 .
$$

Furthermore, if $V^{2}(\bar{u}, u) \cap L^{2}\left(0, T ; H_{1}\right) \neq \emptyset$, then for any $w_{T} \in \mathrm{cl} R^{L(2)}$ satisfying (2.22), there exist trajectorycontrol pairs $\left\{\left(x_{n}, u_{n}\right)\right\}_{n \in \mathbb{N}}$ of $(1.1)$ and $\delta_{n} \rightarrow 0+$ such that $h\left(x_{n}(T)\right)=0, \lim _{n \rightarrow \infty}\left|u_{n}-\bar{u}\right|_{L^{1}\left(0, T ; H_{1}\right)}=0$ and

$$
\lim _{n \rightarrow \infty} \frac{\left|x_{n}(T)-\bar{x}(T)-\delta_{n} y(T)-\delta_{n}^{2} w_{T}\right|_{H}}{\delta_{n}^{2}}=0 .
$$

Lemma 3.19. Assume (S1)-(S4). Let $(\bar{x}, \bar{u})$ be a trajectory-control pair of $(1.1)$ with $h(\bar{x}(T))=0$ satisfying (2.17) and $(y, u) \in \Xi$ be a trajectory-control pair of (2.6) satisfying $(2.11)$ and such that $h_{x}(\bar{x}(T))(y(T))=0$. 
Then for every trajectory-control pair $(w, v) \in \Xi$ of (2.13) such that $(2.22)$ holds true with $w_{T}=w(T)$ there exists $c>0$ and trajectory-control pairs $\left\{\left(x_{\delta}, u_{\delta}\right)\right\}_{\delta>0}$ of (1.1) satisfying $\left|u_{\delta}-\bar{u}\right|_{L^{1}\left(0, T ; H_{1}\right)} \leq c \delta$,

$$
h\left(x_{\delta}(T)\right)=0
$$

and

$$
\lim _{\delta \rightarrow 0+} \frac{\left|x_{\delta}-\bar{x}-\delta y-\delta^{2} w\right|_{C([0, T] ; X)}}{\delta^{2}}=0
$$

Furthermore, if $V^{2}(\bar{u}, u) \cap L^{2}\left(0, T ; H_{1}\right) \neq \emptyset$, then for any $w_{T} \in \operatorname{cl} R^{L(2)}$ satisfying (2.22), there exist trajectorycontrol pairs $\left\{\left(x_{n}, u_{n}\right)\right\}_{n \in \mathbb{N}}$ of $(1.1)$ and $\delta_{n} \rightarrow 0+$ such that $h\left(x_{n}(T)\right)=0, \lim _{n \rightarrow \infty}\left|u_{n}-\bar{u}\right|_{L^{1}\left(0, T ; H_{1}\right)}=0$ and

$$
\lim _{n \rightarrow \infty} \frac{\left|x_{n}(T)-\bar{x}(T)-\delta_{n} y(T)-\delta_{n}^{2} w_{T}\right|_{X}}{\delta_{n}^{2}}=0 .
$$

Proposition 3.20. Let $\tilde{u} \in \mathcal{U}$ and $u, v:[0, T] \rightarrow H_{1}$ be measurable and such that

(i) for some measurable $c:[0, T] \rightarrow \mathbb{R}_{+}, \rho_{0}>0$ and for any $\rho \in\left(0, \rho_{0}\right] \operatorname{dist}(\tilde{u}(t)+\rho u(t), U) \leq \rho^{2} c(t)$ for a.e. $t \in[0, T]$

(ii) $v(t) \in T_{U}^{b(2)}(\tilde{u}(t), u(t))$ for a.e. $t \in[0, T]$.

Then for any $\rho \in\left(0, \rho_{0}\right]$, there exists a measurable $v_{\rho}:[0, T] \rightarrow H_{1}$ satisfying

(1) $\left|v_{\rho}(t)\right| \leq 2|v(t)|+2 \rho+c(t)$ for a.e. $t \in[0, T]$;

(2) $\tilde{u}(t)+\rho u(t)+\rho^{2} v_{\rho}(t) \in U$ for a.e. $t \in[0, T]$;

(3) $\lim _{\rho \rightarrow 0+} v_{\rho}(t)=v(t)$ for a.e. $t \in[0, T]$.

In particular, if $v \in L^{2}\left(0, T ; H_{1}\right)$ and $c \in L^{2}(0, T)$, then $\lim _{\rho \rightarrow 0+}\left|v_{\rho}-v\right|_{L^{2}\left(0, T ; H_{1}\right)}=0$.

Proof. Let $\rho \in\left(0, \rho_{0}\right]$. Put

$$
a_{\rho}(t) \triangleq \operatorname{dist}\left(\tilde{u}(t)+\rho u(t)+\rho^{2} v(t), U\right)
$$

Then $a_{\rho}$ is measurable. Further, by Lemmas 3.5 and 3.6, there exists a measurable $y_{\rho}:[0, T] \rightarrow U$ such that

$$
a_{\rho}(t) \leq\left|\tilde{u}(t)+\rho u(t)+\rho^{2} v(t)-y_{\rho}(t)\right|_{H_{1}} \leq a_{\rho}(t)+\rho^{3} \quad \text { for a.e. } t \in[0, T] .
$$

Similarly, there exists a measurable $z_{\rho}:[0, T] \rightarrow U$ such that

$$
\left|\tilde{u}(t)+\rho u(t)-z_{\rho}(t)\right|_{H_{1}} \leq \operatorname{dist}(\tilde{u}(t)+\rho u(t), U)+\rho^{3} \leq \rho^{2} c(t)+\rho^{3} \quad \text { for a.e. } t \in[0, T] .
$$

This, together with (3.17), implies that

$$
a_{\rho}(t) \leq\left|\tilde{u}(t)+\rho u(t)+\rho^{2} v(t)-z_{\rho}(t)\right|_{H_{1}} \leq \rho^{2} c(t)+\rho^{2}|v(t)|_{H_{1}}+\rho^{3} \text { for a.e. } t \in[0, T] .
$$

Consequently,

$$
\frac{a_{\rho}(t)}{\rho^{2}} \leq c(t)+|v(t)|_{H_{1}}+\rho \quad \text { for a.e. } t \in(0, T)
$$


Next, define $v_{\rho}:[0, T] \rightarrow H_{1}$ by

$$
v_{\rho}(t) \triangleq \frac{y_{\rho}(t)-\rho u(t)-\tilde{u}(t)}{\rho^{2}}
$$

Then we have that

$$
\left|v(t)-v_{\rho}(t)\right|_{H_{1}} \leq \frac{a_{\rho}(t)}{\rho^{2}}+\rho \quad \text { for a.e. } t \in[0, T]
$$

Therefore, for a.e. $t \in[0, T]$,

$$
\left|v_{\rho}(t)\right|_{H_{1}} \leq 2|v(t)|_{H_{1}}+c(t)+2 \rho .
$$

Further, it follows directly from the definition of $v_{\rho}$ that

$$
\tilde{u}(t)+\rho u(t)+\rho^{2} v_{\rho}(t) \in U \text { for a.e. } t \in[0, T] .
$$

Finally, since for a.e. $t \in[0, T], v(t) \in T_{U}^{b(2)}(\tilde{u}(t), u(t))$, we have that

$$
\lim _{\rho \rightarrow 0+} \frac{a_{\rho}(t)}{\rho^{2}}=0 \quad \text { for a.e. } t \in[0, T] .
$$

Proof of Lemma 3.18. By Proposition 3.20, we know that for every $\delta>0$ there exists $\tilde{u}_{\delta} \in \mathcal{U}$ such that

$$
\lim _{\delta \rightarrow 0+} \frac{\tilde{u}_{\delta}-\bar{u}-\delta u}{\delta^{2}}=v \text { in } L^{2}\left(0, T ; H_{1}\right) .
$$

Denote by $\tilde{x}_{\delta}$ the solution of $(1.1)$ corresponding to $\tilde{u}_{\delta}$. It follows from (3.18) that

$$
\begin{gathered}
\left|\tilde{u}_{\delta}-\bar{u}\right|_{L^{2}\left(0, T ; H_{1}\right)}=O(\delta), \\
\left|\tilde{u}_{\delta}-\bar{u}-\delta u\right|_{L^{2}\left(0, T ; H_{1}\right)}=O\left(\delta^{2}\right)
\end{gathered}
$$

and

$$
\left|\tilde{u}_{\delta}-\bar{u}-\delta u-\delta^{2} v\right|_{L^{2}\left(0, T ; H_{1}\right)}=o\left(\delta^{2}\right) .
$$

Then we have that for some $C>0$,

$$
\begin{aligned}
& \left|\tilde{x}_{\delta}(t)-\bar{x}(t)\right|_{H} \\
= & \left|\int_{0}^{t} e^{A(t-s)} f\left(s, \tilde{x}_{\delta}(s), \tilde{u}_{\delta}(s)\right) \mathrm{d} s-\int_{0}^{t} e^{A(t-s)} f(s, \bar{x}(s), \bar{u}(s)) \mathrm{d} s\right|_{H} \\
\leq & \left|\int_{0}^{t} e^{A(t-s)} f_{x}[s]\left(\tilde{x}_{\delta}(s)-\bar{x}(s)\right) \mathrm{d} s\right|_{H}+\left|\int_{0}^{t} e^{A(t-s)} f_{u}[s]\left(\tilde{u}_{\delta}(s)-\bar{u}(s)\right) \mathrm{d} s\right|_{H} \\
& +\frac{1}{2}\left|\int_{0}^{t} e^{A(t-s)}\left[\int_{0}^{1}(1-\theta) f_{x x}\left(s, \bar{x}(s)+\theta\left(\tilde{x}_{\delta}(s)-\bar{x}(s)\right), \bar{u}(s)\right) \mathrm{d} \theta\right]\left(\tilde{x}_{\delta}(s)-\bar{x}(s), \tilde{x}_{\delta}(s)-\bar{x}(s)\right) \mathrm{d} s\right|_{H}
\end{aligned}
$$




$$
\begin{aligned}
& +\frac{1}{2}\left|\int_{0}^{t} e^{A(t-s)}\left[\int_{0}^{1}(1-\theta) f_{x u}\left(s, \bar{x}(s)+\theta\left(\tilde{x}_{\delta}(s)-\bar{x}(s)\right), \bar{u}(s)\right) \mathrm{d} \theta\right]\left(\tilde{x}_{\delta}(s)-\bar{x}(s), \tilde{u}_{\delta}(s)-\bar{u}(s)\right) \mathrm{d} s\right|_{H} \\
& +\frac{1}{2}\left|\int_{0}^{t} e^{A(t-s)}\left[\int_{0}^{1}(1-\theta) f_{x u}\left(s, \bar{x}(s), \bar{u}(s)+\theta\left(\tilde{u}_{\delta}(s)-\bar{u}(s)\right)\right) \mathrm{d} \theta\right]\left(\tilde{x}_{\delta}(s)-\bar{x}(s), \tilde{u}_{\delta}(s)-\bar{u}(s)\right) \mathrm{d} s\right|_{H} \\
& +\frac{1}{2}\left|\int_{0}^{t} e^{A(t-s)}\left[\int_{0}^{1}(1-\theta) f_{u u}\left(s, \bar{x}(s), \bar{u}(s)+\theta\left(\tilde{u}_{\delta}(s)-\bar{u}(s)\right)\right) \mathrm{d} \theta\right]\left(\tilde{u}_{\delta}(s)-\bar{u}(s), \tilde{u}_{\delta}(s)-\bar{u}(s)\right) \mathrm{d} s\right|_{H} \\
& \leq C\left(\int_{0}^{t}\left|\tilde{x}_{\delta}(s)-\bar{x}(s)\right|_{H} \mathrm{~d} s+\delta\right) .
\end{aligned}
$$

This, together with Gronwall's inequality, implies that

$$
\left|\tilde{x}_{\delta}-\bar{x}\right|_{C([0, T] ; H)} \leq C \delta
$$

Similarly, from (3.20) and the above inequalities, we can deduce that

$$
\left|\tilde{x}_{\delta}-\bar{x}-\delta y\right|_{C([0, T] ; H)} \leq C \delta^{2}
$$

From (3.19) and (3.22) it follows that

$$
\begin{aligned}
& \left|\tilde{x}_{\delta}(t)-\bar{x}(t)-\delta y(t)-\delta^{2} w(t)\right|_{H} \\
& =\mid \int_{0}^{t} e^{A(t-s)} f\left(s, \tilde{x}_{\delta}(s), \tilde{u}_{\delta}(s)\right) \mathrm{d} s-\int_{0}^{t} e^{A(t-s)} f(s, \bar{x}(s), \bar{u}(s)) \mathrm{d} s \\
& \quad-\delta \int_{0}^{t} e^{A(t-s)}\left(f_{x}[s] y(s)+f_{u}[s] u(s)\right) \mathrm{d} s-\delta^{2} \int_{0}^{t} e^{A(t-s)}\left(f_{x}[s] w(s)+f_{u}[s] v(s)\right) \mathrm{d} s \\
& \quad-\left.\frac{\delta^{2}}{2} \int_{0}^{t} e^{A(t-s)}\left[f_{x x}[s](y(s), y(s))+2 f_{x u}[s](y(s), u(s))+f_{u u}[s](u(s), u(s))\right] \mathrm{d} s\right|_{H} \\
& =\mid \int_{0}^{t} e^{A(t-s)} f_{x}[s]\left(\tilde{x}_{\delta}(s)-\bar{x}(s)-\delta y(s)-\delta^{2} w(s)\right) \mathrm{d} s \\
& \quad+\int_{0}^{t} e^{A(t-s)} f_{u}[s]\left(\tilde{u}_{\delta}(s)-\bar{u}(s)-\delta u(s)-\delta^{2} v(s)\right) \mathrm{d} s \\
& \quad+\frac{1}{2} \int_{0}^{t} e^{A(t-s)}\left[f_{x x}[s]\left(\tilde{x}_{\delta}(s)-\bar{x}(s), \tilde{x}_{\delta}(s)-\bar{x}(s)\right)+2 f_{x u}[s]\left(\tilde{x}_{\delta}(s)-\bar{x}(s), \tilde{u}_{\delta}(s)-\bar{u}(s)\right)\right. \\
& \quad+\left.\frac{\delta^{2}}{2} \int_{0}^{t} e^{A(t-s)}\left[f_{x x}[s](y(s), y(s))+2 f_{x u}[s](y(s), u(s))+f_{u u}[s](u(s), u(s))\right] \mathrm{d} s\right|_{H}+o_{t}\left(\delta^{2}\right)
\end{aligned}
$$

with $\sup _{t \in[0, T]}\left|o_{t}\left(\delta^{2}\right)\right|=o\left(\delta^{2}\right)$.

By (3.22) and (3.23), we see that for every $t \in[0, T]$,

$$
\begin{aligned}
& \int_{0}^{t}\left|f_{x x}[s]\left(\tilde{x}_{\delta}(s)-\bar{x}(s), \tilde{x}_{\delta}(s)-\bar{x}(s)\right)-\delta^{2} f_{x x}[s](y(s), y(s))\right|_{H} \mathrm{~d} s \\
& =\int_{0}^{t}\left|f_{x x}[s]\left(\tilde{x}_{\delta}(s)-\bar{x}(s), \tilde{x}_{\delta}(s)-\bar{x}(s)-\delta y(s)\right)+f_{x x}[s]\left(\delta y(s), \tilde{x}_{\delta}(s)-\bar{x}(s)-\delta y(s)\right)\right|_{H} \mathrm{~d} s \\
& \leq o\left(\delta^{2}\right) .
\end{aligned}
$$


Similarly, we can show that

$$
\begin{aligned}
& \mid \int_{0}^{t}\left[2 f_{x u}[s]\left(\tilde{x}_{\delta}(s)-\bar{x}(s), \tilde{u}_{\delta}(s)-\bar{u}(s)\right)+f_{u u}[s]\left(\tilde{u}_{\delta}(s)-\bar{u}(s), \tilde{u}_{\delta}(s)-\bar{u}(s)\right)\right] \mathrm{d} s \\
& \quad-\left.\delta^{2} \int_{0}^{t}\left[2 f_{x u}[s](y(s), u(s))+f_{u u}[s](u(s), u(s))\right] \mathrm{d} s\right|_{H} \leq o\left(\delta^{2}\right) .
\end{aligned}
$$

Combining (3.21), (3.24), (3.25) and (3.26), we obtain that

$$
\left|\tilde{x}_{\delta}(t)-\bar{x}(t)-\delta y(t)-\delta^{2} w(t)\right|_{H} \leq C \int_{0}^{t}\left|\tilde{x}_{\delta}(s)-\bar{x}(s)-\delta y(s)-\delta^{2} w(s)\right|_{H} \mathrm{~d} s+o\left(\delta^{2}\right) .
$$

This and Gronwall's inequality imply that

$$
\left|\tilde{x}_{\delta}-\bar{x}-\delta y-\delta^{2} w\right|_{C([0, T] ; H)}=o\left(\delta^{2}\right)
$$

which yields

$$
\lim _{\delta \rightarrow 0+} \frac{\tilde{x}_{\delta}-\bar{x}-\delta y}{\delta^{2}}=w \text { in } C([0, T] ; H) .
$$

Then by the choice of $y$ and $w, h\left(\tilde{x}_{\delta}(T)\right)=o\left(\delta^{2}\right)$. By Theorem 3.12, for every $\delta>0$, there exists a trajectorycontrol pair $\left(x_{\delta}, u_{\delta}\right)$ of $(1.1)$ such that

$$
\left|\tilde{u}_{\delta}-u_{\delta}\right|_{L^{1}\left(0, T ; H_{1}\right)}=o\left(\delta^{2}\right)
$$

and $h\left(x_{\delta}(T)\right)=0$. Thus,

$$
\left|u_{\delta}-\bar{u}\right|_{L^{1}\left(0, T ; H_{1}\right)} \leq\left|u_{\delta}-\tilde{u}_{\delta}\right|_{L^{1}\left(0, T ; H_{1}\right)}+\left|\tilde{u}_{\delta}-\bar{u}\right|_{L^{1}\left(0, T ; H_{1}\right)} \leq C \delta+o\left(\delta^{2}\right) .
$$

Similarly to the proof of (3.22), we can get that

$$
\left|\tilde{x}_{\delta}-x_{\delta}\right|_{C([0, T] ; H)}=o\left(\delta^{2}\right)
$$

This, together with (3.27), implies that

$$
\left|x_{\delta}-\bar{x}-\delta y-\delta^{2} w\right|_{C([0, T] ; H)}=o\left(\delta^{2}\right)
$$

Consequently,

$$
\lim _{\delta \rightarrow 0+} \frac{x_{\delta}-\bar{x}-\delta y}{\delta^{2}}=w \text { in } C([0, T] ; H)
$$

To prove the last statement, consider trajectory-control pairs $\left(w_{n}, v_{n}\right)$ of $(2.13)$ such that $\lim _{n \rightarrow \infty} w_{n}(T)=w_{T}$. Since $V^{2}(\bar{u}, u) \cap L^{2}\left(0, T ; H_{1}\right) \neq \emptyset$, we may assume that $\left(w_{n}, v_{n}\right) \in \Xi$. 
Fix $n$ and let $\left(\tilde{x}_{\delta}, \tilde{u}_{\delta}\right)$ be as at the beginning of the proof with $w$ replaced by $w_{n}$. In particular, $\lim _{\delta \rightarrow 0+} \mid \tilde{u}_{\delta}-$ $\left.\bar{u}\right|_{L^{1}\left(0, T ; H_{1}\right)}=0$ and

$$
\lim _{\delta \rightarrow 0+} \frac{\left|\tilde{x}_{\delta}-\bar{x}-\delta y-\delta^{2} w_{n}\right|_{C([0, T] ; H)}}{\delta^{2}}=0
$$

On the other hand, setting

$$
\varepsilon_{n}=\left|2 h_{x}(\bar{x}(T))\left(w_{n}(T)\right)+h_{x x}(\bar{x}(T))(y(T), y(T))\right|+\frac{1}{n},
$$

we know that $\lim _{n \rightarrow \infty} \varepsilon_{n}=0$. By the choice of $y$, for all $\delta>0$ sufficiently small,

$$
\left|h\left(\tilde{x}_{\delta}(T)\right)\right| \leq \varepsilon_{n} \delta^{2}
$$

By Theorem 3.12 there exists $c>0$ independent of $n$ and $\bar{\delta}_{n}>0$, such that for every $\delta \in\left(0, \bar{\delta}_{n}\right]$, we can find a trajectory-control pair $\left(x_{\delta}, u_{\delta}\right)$ of $(1.1)$ such that

$$
\left|\tilde{u}_{\delta}-u_{\delta}\right|_{L^{1}\left(0, T ; H_{1}\right)} \leq c \varepsilon_{n} \delta^{2}
$$

and $h\left(x_{\delta}(T)\right)=0$. Then, by the Gronwall inequality, for a constant $C>0$ independent of $n$, we have $\mid x_{\delta}-$ $\left.\tilde{x}_{\delta}\right|_{C([0, T] ; H)} \leq C \varepsilon_{n} \delta^{2}$. Taking $\bar{\delta}_{n}$ smaller and keeping the same notation, we may assume that

$$
\frac{\left|\tilde{x}_{\delta}-\bar{x}-\delta y-\delta^{2} w_{n}\right|_{C([0, T] ; H)}}{\delta^{2}} \leq \varepsilon_{n}
$$

for all $\delta \in\left(0, \bar{\delta}_{n}\right]$. Then

$$
\frac{\left|x_{\delta}-\bar{x}-\delta y-\delta^{2} w_{n}\right|_{C([0, T] ; H)}}{\delta^{2}} \leq(C+1) \varepsilon_{n}
$$

for all $\delta \in\left(0, \bar{\delta}_{n}\right]$. Consider next any $\delta_{n} \in\left(0, \bar{\delta}_{n}\right]$ such that $\delta_{n} \rightarrow 0+$ and define $\left(x_{n}, u_{n}\right)=\left(x_{\delta_{n}}, u_{\delta_{n}}\right)$. Then

$$
\frac{\left|x_{n}(T)-\bar{x}(T)-\delta_{n} y(T)-\delta_{n}^{2} w_{T}\right|}{\delta_{n}^{2}} \leq(C+1) \varepsilon_{n}+\left|w_{n}(T)-w_{T}\right|_{H}
$$

The proof is complete

The proof of Lemma 3.19 is very similar to that of Lemma 3.18. One only needs to use the smoothing effect to compensate the loss of the regularity of the derivatives of $f, h$, etc. Indeed, for any $x_{0} \in H$, the solution $x(t) \in X$ for $t>0$. Hence, for $t>0$, the terms such as $\left|f_{x}(t, x(t), u)\right|_{\mathcal{L}(X ; H)},\left|f_{x x}(t, x(t), u)\right|_{\mathcal{L}(X, X ; H)}$ make sense. We omit the details.

\section{Proofs of the MAin ReSults}

We borrow some ideas from $[8,10]$ to prove Theorem 2.5. The key point is to find "admissible perturbations" of the control (perturbation of the local minimizer which still fulfill the endpoints constraints) using Lemma 3.18, the second order linearization of our control system and second order linearizations of the end-point constraints. Then we write a variational inequality satisfied by these perturbations and derive a dual expression of this variational inequality by using the separation theorem. To get the above variational inequality we need a 
Mangasarian-Fromovitz like constraint qualification. When it fails to hold, again, by using the same kind of arguments based on the separation theorem the necessary conditions follow in the abnormal form.

Proof of Theorem 2.5. By (2.18), the mapping $h_{x}(\bar{x}(T))$ is surjective. Thus, $h_{x}(\bar{x}(T))^{*}$ is injective and

$$
\left(\operatorname{ker} h_{x}(\bar{x}(T))\right)^{\perp}=\operatorname{Im} h_{x}(\bar{x}(T))^{*}
$$

Fix $(y, u) \in \mathcal{C}(\bar{x}, \bar{u})$ with $V^{2}(\bar{u}, u) \cap L^{2}\left(0, T ; H_{1}\right) \neq \emptyset$. By Lemma 3.11, we obtain that

$$
\mathcal{R}+R^{L(2)}=R^{L(2)}
$$

Put

$$
Q_{j}=\left\{\eta \in H \mid g_{j, x}(\bar{x}(T))(\eta)+\frac{1}{2} g_{j, x x}(\bar{x}(T))(y(T), y(T))<0\right\}, \quad j \in \mathcal{I}_{g} \cup\{0\}
$$

and observe that if for some $\ell \in \mathcal{I}_{g} \cup\{0\}, Q_{\ell}=\emptyset$, then for all $\eta \in H$,

$$
g_{\ell, x}(\bar{x}(T))(\eta)+\frac{1}{2} g_{\ell, x x}(\bar{x}(T))(y(T), y(T)) \geq 0
$$

This implies that

$$
g_{\ell, x}(\bar{x}(T))=0, \quad g_{\ell, x x}(\bar{x}(T))(y(T), y(T)) \geq 0 .
$$

In this case, set $\beta=0, \alpha_{\ell}=1$ and $\alpha_{j}=0$ for $j \neq \ell$. Then $(\alpha, 0,0) \in \Lambda(\bar{x}, \bar{u})$ and (2.19) is verified with $p(\cdot)=0$.

Now we only need to deal with the case when $Q_{j} \neq \emptyset$ for every $j \in \mathcal{I}_{g} \cup\{0\}$.

From (4.3), we see that if $g_{j, x}(\bar{x}(T))=0$, then $Q_{j}=H$. Put

$$
\mathcal{I} \triangleq\left\{j \in \mathcal{I}_{g} \mid g_{j, x}(\bar{x}(T)) \neq 0, g_{j, x}(\bar{x}(T))(y(T))=0\right\}
$$

In what follows, for convenience, if $\mathcal{I}=\emptyset$, then we set $\bigcap_{j \in \mathcal{I}} Q_{j}=H$.

The remaining proof is quite long and is divided into three steps. It is well known that the set $\mathrm{cl} R^{L(2)}$ is convex (it can be verified similarly to the end of the proof of Lemma 3.15, where we have shown that $\operatorname{cl}\left(h_{x}(\bar{x}(T)) R^{L, \bar{u}}\right)$ is convex).

Step 1. Assume that $\left(\bigcap_{j \in \mathcal{I}} Q_{j}\right) \bigcap \Theta \bigcap \mathrm{cl} R^{L(2)} \neq \emptyset$ and let $w_{T} \in\left(\bigcap_{j \in \mathcal{I}} Q_{j}\right) \bigcap \Theta \bigcap \operatorname{cl} R^{L(2)}$ (recall (2.14) for the definition of $\Theta$ ). Let $\left(x_{n}, u_{n}\right)$ and $\delta_{n} \rightarrow 0+$ be as in the last statement of Lemma 3.18. If $j \notin \mathcal{I}_{g}$, then for all large $n, x_{n}(T) \in Q_{j}$ and therefore $g_{j}\left(x_{n}(T)\right) \leq 0$. Also if for some $j \in \mathcal{I}_{g}$ we have $g_{j, x}(\bar{x}(T))(y(T))<0$, then for all large $n$

$$
g_{j}\left(x_{n}(T)\right)=g_{j}(\bar{x}(T))+\delta_{n} g_{j, x}(\bar{x}(T))(y(T))+o\left(\delta_{n}\right)<0
$$

Finally, if $g_{j, x}(\bar{x}(T))(y(T))=0$ for some $j \in \mathcal{I}_{g}$, then for all large $n$

$$
g_{j}\left(x_{n}(T)\right)=g_{j}(\bar{x}(T))+\delta_{n}^{2}\left(g_{j, x}(\bar{x}(T))\left(w_{T}\right)+\frac{1}{2} g_{j, x x}(\bar{x}(T))(y(T), y(T))\right)+o\left(\delta_{n}^{2}\right)<0
$$


Consequently, $\left(x_{n}, u_{n}\right)$ is admissible whenever $n$ is sufficiently large. It follows from the inequality $g_{0, x}(\bar{x}(T))(y(T)) \leq 0$ that

$$
\begin{aligned}
0 \leq & \lim _{n \rightarrow \infty} \frac{g_{0}\left(x_{n}(T)\right)-g_{0}(\bar{x}(T))}{\delta_{n}^{2}} \\
\leq & \lim _{n \rightarrow \infty} \frac{g_{0, x}(\bar{x}(T))\left(x_{n}(T)-\bar{x}(T)-\delta_{n} y(T)\right)}{\delta_{n}^{2}} \\
& +\frac{1}{2} \lim _{n \rightarrow \infty} g_{0, x x}(\bar{x}(T))\left(\frac{x_{n}(T)-\bar{x}(T)}{\delta_{n}}, \frac{x_{n}(T)-\bar{x}(T)}{\delta_{n}}\right) \\
= & g_{0, x}(\bar{x}(T))\left(w_{T}\right)+\frac{1}{2} g_{0, x x}(\bar{x}(T))(y(T), y(T)) .
\end{aligned}
$$

If $g_{0, x}(\bar{x}(T))=0$, set $\alpha_{0}=1, \alpha_{j}=0$ for $j \neq 0$ and $\beta=0, p(\cdot)=0$. Then, by the above inequality, the conclusion of Theorem 2.5 holds with this choice of multipliers. From now on we assume that $g_{0, x}(\bar{x}(T)) \neq 0$.

If $\mathcal{I} \neq \emptyset$, let $\mathcal{I}=\left\{j_{1}, \ldots, j_{\gamma}\right\}$, where $1 \leq \gamma \leq r$. The above string of inequalities implies that the convex set

$$
\Gamma \triangleq\left\{\left(q_{0}-\kappa, q_{1}-\kappa, \ldots, q_{\gamma}-\kappa, \theta-\kappa\right) \mid \kappa \in \operatorname{cl} R^{L(2)}, \theta \in \Theta, q_{0} \in Q_{0}, q_{\ell} \in Q_{j_{\ell}}, \ell=1, \ldots, \gamma\right\}
$$

does not contain 0 . By (H4), we know that $\Gamma$ has interior points in the product space $\prod_{j=0}^{\gamma} E_{j} \times \widetilde{H}$, where $E_{j}=H$ for all $j=0, \ldots, \gamma$. By the separation theorem for convex sets, there exists a nonzero bounded linear functional $\rho$ on $\prod_{j=0}^{\gamma} E_{j} \times \widetilde{H}$ such that

$$
\begin{aligned}
& \rho\left(q_{0}-\kappa, q_{1}-\kappa, \ldots, q_{\gamma}-\kappa, \theta-\kappa\right) \geq 0 \\
& \quad \forall \kappa \in \operatorname{cl} R^{L(2)}, \theta \in \Theta, q_{0} \in Q_{0}, q_{\ell} \in Q_{j_{\ell}}, \quad \ell=1, \ldots, \gamma .
\end{aligned}
$$

Define an extension $\tilde{\rho}$ of $\rho$ as follows:

$$
\tilde{\rho}\left(\zeta_{0}, \ldots, \zeta_{\gamma}, \zeta_{\gamma+1}\right)=\rho\left(\zeta_{0}, \ldots, \zeta_{\gamma}, \mathbb{P}_{\widetilde{H}} \zeta_{\gamma+1}\right), \quad \forall\left(\zeta_{0}, \ldots, \zeta_{\gamma}, \zeta_{\gamma+1}\right) \in \prod_{j=0}^{\gamma} E_{j} \times H,
$$

where $\mathbb{P}_{\widetilde{H}}$ denotes the orthogonal projection from $H$ to $\widetilde{H}$. Then we have

$$
\begin{aligned}
& \tilde{\rho}\left(q_{0}-\kappa, q_{1}-\kappa, \ldots, q_{\gamma}-\kappa, \theta-\kappa\right) \geq 0, \\
& \quad \forall \kappa \in \operatorname{cl} R^{L(2)}, \theta \in \Theta, q_{0} \in Q_{0}, q_{\ell} \in Q_{j_{\ell}}, \quad \ell=1, \ldots, \gamma .
\end{aligned}
$$

By the Riesz representation theorem, there exist $\zeta, \zeta_{j} \in H(j \in \mathcal{I} \cup\{0\})$, not vanishing simultaneously, such that for every $\kappa \in \operatorname{cl} R^{L(2)}$,

$$
\sum_{j \in \mathcal{I} \cup\{0\}} \inf _{q_{j} \in Q_{j}}\left\langle\zeta_{j}, q_{j}-\kappa\right\rangle_{H}+\inf _{\theta \in \Theta}\langle\zeta, \theta-\kappa\rangle_{H} \geq 0 .
$$

By taking $p_{1}=-\sum_{j \in \mathcal{I} \cup\{0\}} \zeta_{j}-\zeta$, we have

$$
\sum_{j \in \mathcal{I} \cup\{0\}} \zeta_{j}+\zeta+p_{1}=0
$$


and

$$
\sum_{j \in \mathcal{I} \cup\{0\}} \inf _{q_{j} \in Q_{j}}\left\langle\zeta_{j}, q_{j}\right\rangle_{H}+\inf _{\theta \in \Theta}\langle\zeta, \theta\rangle_{H}+\inf _{k \in R^{L(2)}}\left\langle p_{1}, k\right\rangle_{H} \geq 0 .
$$

It follows from (4.6) that

$$
\inf \left\{\langle\zeta, \kappa\rangle_{H} \mid \kappa \in \operatorname{ker} h_{x}(\bar{x}(T))\right\}>-\infty
$$

implying that $\zeta \in \operatorname{ker} h_{x}(\bar{x}(T))^{\perp}$. Hence, by (4.1), we get that

$$
\zeta=h_{x}(\bar{x}(T))^{*} q_{1} \text { for some } q_{1} \in H_{2} .
$$

Set $\beta=-q_{1}$. Similarly, by the definition of $Q_{j}$, for every $j \in \mathcal{I} \cup\{0\}$, we have

$$
\zeta_{j} \in\left(\operatorname{ker} g_{j, x}(\bar{x}(T))\right)^{\perp}=\operatorname{Im} g_{j, x}(\bar{x}(T))^{*} .
$$

Consequently,

$$
\zeta_{j}=\lambda_{j} g_{j, x}(\bar{x}(T)) \text { for some } \lambda_{j} \in \mathbb{R}
$$

We next observe that for all large $\lambda>0,-\lambda g_{j, x}(\bar{x}(T)) \in Q_{j}$. By (4.6), we have that for some $c>0$

$$
\inf _{\lambda>c}\left\langle\lambda_{j} g_{j, x}(\bar{x}(T)),-\lambda g_{j, x}(\bar{x}(T))\right\rangle_{H}=\inf _{\lambda>c}\left(-\lambda \lambda_{j}\left|g_{j, x}(\bar{x}(T))\right|_{H}^{2}\right)>-\infty .
$$

Recalling that $\left|g_{j, x}(\bar{x}(T))\right|_{H}^{2}>0$, we deduce that $\lambda_{j} \leq 0$. By the very definition of $Q_{j}$, for every $j \in \mathcal{I} \cup\{0\}$,

$$
\sup _{\kappa \in Q_{j}} g_{j, x}(\bar{x}(T))(\kappa)=-\frac{1}{2} g_{j, x x}(\bar{x}(T))(y(T), y(T)) .
$$

Therefore,

$$
\inf _{\kappa \in Q_{j}} \zeta_{j}(\kappa)=\frac{\left|\lambda_{j}\right|}{2} g_{j, x x}(\bar{x}(T))(y(T), y(T))
$$

Set

$$
\alpha_{j}= \begin{cases}\left|\lambda_{j}\right|, & \text { if } j \in \mathcal{I} \cup\{0\}, \\ 0, & \text { if } j \in\{1, \ldots, r\} \backslash \mathcal{I}\end{cases}
$$

By (4.5),

$$
p_{1}=\sum_{j=0}^{r} \alpha_{j} g_{j, x}(\bar{x}(T))+h_{x}(\bar{x}(T))^{*} \beta .
$$

Since $\zeta^{*}$ and $\left\{\zeta_{j}^{*}\right\}_{j \in \mathcal{I} \cup\{0\}}$ do not vanish simultaneously, we have $(\alpha, \beta) \neq 0$. Denote by $p(\cdot)$ the solution to the adjoint equation (2.2) with the final datum $p_{T}=p_{1}$. It follows from (4.6) that $\inf _{\kappa \in R^{L(2)}} p(T)(\kappa)$ is bounded 
from below. Since $\mathcal{R}$ is a cone, we deduce from (4.2) and (4.6) that

$$
\inf _{\kappa \in \mathcal{R}} p(T)(\kappa) \geq 0 \text {. }
$$

Let $(z, \pi)$ be a trajectory-control pair of $(2.20)$. Then

$$
\langle p(T), z(T)\rangle_{H}=\int_{0}^{T}\left\langle p(t), f_{u}[t] \pi(t)\right\rangle_{H} \mathrm{~d} t \geq 0 .
$$

From Lemmas 3.10 and 3.5 , using that $\mathcal{C}_{U}(\bar{u}(t))$ is a cone, we deduce that

$$
\inf _{\kappa \in C_{U}(\bar{u}(t))}\left\langle p(t), f_{u}[t] \kappa\right\rangle_{H}=0
$$

Hence $(\alpha, \beta, p) \in \Lambda(\bar{x}, \bar{u})$.

Step 2. Let $(w, v)$ be a trajectory-control pair of (2.13). From (4.6) and the above calculations we obtain

$$
\frac{1}{2}\left\langle h_{x x}(\bar{x}(T))(y(T), y(T)), \beta\right\rangle_{H_{2}}+\sum_{j \in \mathcal{I} \cup\{0\}} \frac{\alpha_{j}}{2} g_{j, x x}(\bar{x}(T))(y(T), y(T))+\langle p(T), w(T)\rangle_{H} \geq 0 .
$$

On the other hand,

$$
\begin{aligned}
\langle & p(T), w(T)\rangle_{H} \\
= & \int_{0}^{T}\left[\left\langle p(t), f_{u}[t] v(t)\right\rangle_{H}+\frac{1}{2}\left(\left\langle p(t), f_{x x}[t](y(t), y(t))\right\rangle_{H}+2\left\langle p(t), f_{x u}[t](y(t), u(t))\right\rangle_{H}\right.\right. \\
& \left.\left.+\left\langle p(t), f_{u u}[t](u(t), u(t))\right\rangle_{H}\right)\right] \mathrm{d} t \\
= & \int_{0}^{T}\left[\mathcal{H}_{u}[t](v(t))+\frac{1}{2}\left(\left\langle\mathcal{H}_{x x}[t] y(t), y(t)\right\rangle_{H}+2 \mathcal{H}_{x u}[t](y(t), u(t))+\mathcal{H}_{u u}[t](u(t), u(t))\right)\right] \mathrm{d} t .
\end{aligned}
$$

This yields

$$
\frac{1}{2} \Omega(y, u, \alpha, \beta, p)+\int_{0}^{T} \mathcal{H}_{u}[t](v(t)) \mathrm{d} t \geq 0 .
$$

Next, for every $\tilde{v} \in V^{2}(\bar{u}, u)$, there exists

$$
\left\{v_{i}\right\}_{i=1}^{\infty} \subset\left\{v \in L^{2}\left(0, T ; H_{1}\right) \mid v(t) \in T_{U}^{b(2)}(\bar{u}(t), u(t)) \text { for a.e. } t \in[0, T]\right\}
$$

such that

$$
\lim _{i \rightarrow \infty} \mathcal{H}_{u}[\cdot]\left(v_{i}(\cdot)\right)=\mathcal{H}_{u}[\cdot](\tilde{v}(\cdot)) \text { in } L^{1}(0, T ; \mathbb{R})
$$

Thus

$$
\frac{1}{2} \Omega(y, u, \alpha, \beta, p)+\inf _{v \in V^{2}(\bar{u}, u)} \int_{0}^{T} \mathcal{H}_{u}[t](v(t)) \mathrm{d} t \geq 0
$$

Then, by an argument similar to end of Section 5 of [10], we can obtain the inequality (2.19). 
Step 3. Assume that $\left(\bigcap_{j \in \mathcal{I}} Q_{j}\right) \cap \Theta \bigcap \mathrm{cl} R^{L(2)}=\emptyset$. Then the set

$$
\Gamma \triangleq\left\{\left(q_{1}-\kappa, \ldots, q_{\gamma}-\kappa, \theta-\kappa\right) \mid \kappa \in \operatorname{cl} R^{L(2)}, \theta \in \Theta, q_{0} \in Q_{0}, q_{\ell} \in Q_{j_{\ell}}, \ell=1, \ldots, \gamma\right\}
$$

does not contain zero. Applying the same separation arguments as in Step 1 and the analysis of Step 2 to this new set $\Gamma$ and $\mathcal{I} \cup\{0\}$ replaced by $\mathcal{I}$ we obtain $(\alpha, \beta, p) \in \Lambda(\bar{x}, \bar{u})$ with $\alpha_{0}=0$ satisfying (2.19).

The proof of Theorem 2.10 is almost the same as the one of Theorem 2.5. One only needs to use the fact that for all $t \in(0, T], \bar{x}(t), y(t), w(t) \in X$. We only focus on the differences in the proof.

Proof of Theorem 2.10. By (2.30), the mapping $h_{x}(\bar{x}(T))$ is surjective. Thus,

$$
\operatorname{Im} h_{x}(\bar{x}(T))^{*}=\left\{\phi \in X^{\prime} \mid \phi(\eta)=0, \forall \eta \in \operatorname{ker} h_{x}(\bar{x}(T))\right\}
$$

Fix $(y, u) \in \mathcal{C}(\bar{x}, \bar{u})$ with $V^{2}(\bar{u}, u) \cap L^{2}\left(0, T ; H_{1}\right) \neq \emptyset$. By Lemma 3.11, we know that (4.2) is satisfied. Define

$$
Q_{j}=\left\{\eta \in X \mid g_{j, x}(\bar{x}(T))(\eta)+\frac{1}{2} g_{j, x x}(\bar{x}(T))(y(T), y(T))<0\right\}, \quad j \in \mathcal{I}_{g} \cup\{0\},
$$

where $\mathcal{I}_{g}$ is as in (2.29). Again, if for some $\ell \in \mathcal{I}_{g} \cup\{0\}, Q_{\ell}=\emptyset$, then it is sufficient to set $\beta=0, \alpha_{\ell}=1$ and $\alpha_{j}=0$ for $j \neq \ell$. Then $(\alpha, 0,0) \in \Lambda_{X}(\bar{x}, \bar{u})$ and $(2.19)$ is verified with $p(\cdot)=0$.

Assume next that $Q_{j} \neq \emptyset$ for every $j \in \mathcal{I}_{g} \cup\{0\}$.

Step 1. From (4.11), we see that if $g_{j, x}(\bar{x}(T))=0$, then $Q_{j}=X$. Put

$$
\mathcal{I} \triangleq\left\{j \in \mathcal{I}_{g} \mid g_{j, x}(\bar{x}(T)) \neq 0, g_{j, x}(\bar{x}(T))(y(T))=0\right\}
$$

Again, if $\mathcal{I}=\emptyset$, then we set $\bigcap_{j \in \mathcal{I}} Q_{j}=X$. that

Let $w_{T} \in\left(\bigcap_{j \in \mathcal{I}} Q_{j}\right) \bigcap \Theta_{X} \bigcap \mathrm{cl}_{X} R^{L(2)}$. In the same way as in Steps 1, 2 of the proof of Theorem 2.5 we show

$$
g_{0, x}(\bar{x}(T))\left(w_{T}\right)+\frac{1}{2} g_{0, x x}(\bar{x}(T))(y(T), y(T)) \geq 0 .
$$

If $g_{0, x}(\bar{x}(T))=0$, set $\alpha_{0}=1, \alpha_{j}=0$ for $j \neq 0$ and $\beta=0, p(\cdot)=0$. Then, by the above inequality, the conclusion of Theorem 2.10 holds with this choice of multipliers. From now on we assume that $g_{0, x}(\bar{x}(T)) \neq 0$.

If $\mathcal{I} \neq \emptyset$, let $\mathcal{I}=\left\{j_{1}, \ldots, j_{\gamma}\right\}$, where $1 \leq \gamma \leq r$. Inequality (4.12) implies that the convex set

$$
\Gamma \triangleq\left\{\left(q_{0}-\kappa, q_{1}-\kappa, \ldots, q_{\gamma}-\kappa, \theta-\kappa\right) \mid \kappa \in \mathrm{cl}_{X} R^{L(2)}, \theta \in \Theta_{X}, q_{0} \in Q_{0}, q_{\ell} \in Q_{j_{\ell}}, \ell=1, \ldots, \gamma\right\}
$$

does not contain 0 . By (S5), $\Gamma$ has interior points in the product space $\prod_{j=0}^{\gamma} X_{j} \times \tilde{X}$, where $X_{j}=X$ for all $j=0, \ldots, \gamma$. By the separation theorem for convex sets, there exists a nonzero bounded linear functional $\rho$ on $\prod_{j=0}^{\gamma} X_{j} \times \widetilde{X}$ such that

$$
\begin{aligned}
& \rho\left(q_{0}-\kappa, q_{1}-\kappa, \ldots, q_{\gamma}-\kappa, \theta-\kappa\right) \geq 0, \\
& \quad \forall \kappa \in \operatorname{cl}_{X} R^{L(2)}, \theta \in \Theta_{X}, q_{0} \in Q_{0}, q_{\ell} \in Q_{j_{\ell}}, \quad \ell=1, \ldots, \gamma .
\end{aligned}
$$


By the Hahn-Banach theorem, $\rho$ has an extension $\tilde{\rho}$ on $\prod_{j=0}^{\gamma} X_{j} \times X$. Then

$$
\begin{aligned}
& \tilde{\rho}\left(q_{0}-\kappa, q_{1}-\kappa, \ldots, q_{\gamma}-\kappa, \theta-\kappa\right) \geq 0, \\
& \quad \forall \kappa \in \operatorname{cl}_{X} R^{L(2)}, \theta \in \Theta_{X}, q_{0} \in Q_{0}, q_{\ell} \in Q_{j_{\ell}}, \quad \ell=1, \ldots, \gamma .
\end{aligned}
$$

By the Riesz representation theorem, there exist $\zeta, \zeta_{j} \in X^{\prime}(j \in \mathcal{I} \cup\{0\})$, not vanishing simultaneously, such that for every $\kappa \in \operatorname{cl}_{X} R^{L(2)}$,

$$
\sum_{j \in \mathcal{I} \cup\{0\}} \inf _{q_{j} \in Q_{j}} \zeta_{j}\left(q_{j}-\kappa\right)+\inf _{\theta \in \Theta_{X}} \zeta(\theta-\kappa) \geq 0
$$

By taking $p_{1}=-\sum_{j \in \mathcal{I} \cup\{0\}} \zeta_{j}-\zeta$, we have

$$
\sum_{j \in \mathcal{I} \cup\{0\}} \zeta_{j}+\zeta+p_{1}=0
$$

and

$$
\sum_{j \in \mathcal{I} \cup\{0\}} \inf _{q_{j} \in Q_{j}} \zeta_{j}\left(q_{j}\right)+\inf _{\theta \in \Theta_{X}} \zeta(\theta)+\inf _{\kappa \in R^{L(2)}} p_{1}(\kappa) \geq 0 .
$$

As in the proof of Theorem 2.5 it follows from (4.15) that $\zeta=h_{x}(\bar{x}(T))^{*} q_{1}$ for some $q_{1} \in H_{2}$. Set $\beta=-q_{1}$. Similarly, $\zeta_{j}=\lambda_{j} g_{j, x}(\bar{x}(T))$ for some $\lambda_{j} \in \mathbb{R}_{-}$. Let $j \in \mathcal{I}$ and $\eta \in X$ be such that $g_{j, x}(\bar{x}(T))(\eta)=\left|g_{j, x}(\bar{x}(T))\right|_{X^{\prime}}$. Then for all large $\lambda>0,-\lambda \eta \in Q_{j}$. By (4.15), we have that for some $c>0$

$$
\inf _{\lambda>c} \lambda_{j} g_{j, x}(\bar{x}(T))(-\lambda \eta)=\inf _{\lambda>c}\left(-\lambda \lambda_{j}\left|g_{j, x}(\bar{x}(T))\right|_{X^{\prime}}\right)>-\infty
$$

and therefore $\lambda_{j} \leq 0$. As before we know that

$$
\inf _{\kappa \in Q_{j}} \zeta_{j}(\kappa)=\frac{\left|\lambda_{j}\right|}{2} g_{j, x x}(\bar{x}(T))(y(T), y(T))
$$

Set

$$
\alpha_{j}= \begin{cases}\left|\lambda_{j}\right|, & \text { if } j \in \mathcal{I} \cup\{0\}, \\ 0, & \text { if } j \in\{1, \ldots, r\} \backslash \mathcal{I} .\end{cases}
$$

By (4.14),

$$
p_{1}=\sum_{j=0}^{r} \alpha_{j} g_{j, x}(\bar{x}(T))+h_{x}(\bar{x}(T))^{*} \beta .
$$

Since $\zeta$ and $\left\{\zeta_{j}\right\}_{j \in \mathcal{I} \cup\{0\}}$ do not vanish simultaneously, we have $(\alpha, \beta) \neq 0$. Denote by $p(\cdot)$ the solution to the adjoint equation (2.2) with the final datum $p_{T}=p_{1}$. It follows from (4.15) that $\inf _{\kappa \in R^{L(2)}} p(T)(\kappa)$ is bounded from below. Since $\mathcal{R}$ is a cone, we deduce from (4.2) and (4.15) that

$$
\inf _{\kappa \in \mathcal{R}} p(T)(\kappa) \geq 0 .
$$


Let $(z, \pi)$ be a trajectory-control pair of $(2.20)$. Then

$$
\langle p(T), z(T)\rangle_{X^{\prime}, X}=\int_{0}^{T}\left\langle p(t), f_{u}[t] \pi(t)\right\rangle_{H} \mathrm{~d} t \geq 0 .
$$

This, together with Lemmas 3.10 and 3.5, implies that $(\alpha, \beta, p) \in \Lambda_{X}(\bar{x}, \bar{u})$.

Step 2. Let $(w, v)$ be a trajectory-control pair of (2.13). From (4.15) and the above calculations we obtain

$$
\frac{1}{2}\left\langle h_{x x}(\bar{x}(T))(y(T), y(T)), \beta\right\rangle_{H_{2}}+\sum_{j \in \mathcal{I} \cup\{0\}} \frac{\alpha_{j}}{2} g_{j, x x}(\bar{x}(T))(y(T), y(T))+\langle p(T), w(T)\rangle_{X^{\prime}, X} \geq 0 .
$$

On the other hand, by assumptions (S1)-(S4),

$$
\begin{aligned}
\langle p(T), w(T)\rangle_{X^{\prime}, X} \\
=\int_{0}^{T}\left[\left\langle p(t), f_{u}[t] v(t)\right\rangle_{H}+\frac{1}{2}\left(\left\langle p(t), f_{x x}[t](y(t), y(t))\right\rangle_{H}+2\left\langle p(t), f_{x u}[t](y(t), u(t))\right\rangle_{H}\right.\right. \\
\left.\left.\quad+\left\langle p(t), f_{u u}[t](u(t), u(t))\right\rangle_{H}\right)\right] \mathrm{d} t \\
=\int_{0}^{T}\left[\mathcal{H}_{u}[t](v(t))+\frac{1}{2}\left(\left\langle\mathcal{H}_{x x}[t] y(t), y(t)\right\rangle_{H}+2 \mathcal{H}_{x u}[t](y(t), u(t))+\mathcal{H}_{u u}[t](u(t), u(t))\right)\right] \mathrm{d} t .
\end{aligned}
$$

This yields

$$
\frac{1}{2} \Omega(y, u, \alpha, \beta, p)+\int_{0}^{T} \mathcal{H}_{u}[t](v(t)) \mathrm{d} t \geq 0
$$

The proof ends in the same way as the one of Theorem 2.5.

Proof of Theorem 2.7. By our assumption, if $j \in \mathcal{I}_{g}$, then $Q_{j} \neq \emptyset$. From the proof of Theorem 2.5 we know that if $g_{0, x}(\bar{x}(T))=0$, then the conclusion of Theorem 2.5 holds with $\alpha_{0}=1, \beta=0, \alpha_{j}=0$ for all $j \neq 0$ and $p(\cdot)=0$.

Now we consider the case of $g_{0, x}(\bar{x}(T)) \neq 0$. From the proof of Theorem 2.5, we only need to show that $\zeta_{0} \neq 0$. Otherwise, let $w_{T}$ be as in the assumptions of our theorem. From (4.5) and (4.6), taking the closure of $R^{L(2)}$ in $H$ we have that

$$
\sum_{j \in \mathcal{I}} \inf _{q_{j} \in Q_{j}}\left\langle\zeta_{j}, q_{j}-w_{T}\right\rangle_{H}+\inf _{\theta \in \Theta}\left\langle\zeta, \theta-w_{T}\right\rangle_{H} \geq 0
$$

This and the choice of $w_{T}$ yield $\zeta_{j}=0$ for every $j \in \mathcal{I}$. Consequently,

$$
p_{1}=-h_{x}(\bar{x}(T))^{*} q_{1} \text { for some } 0 \neq q_{1} \in H_{2} .
$$

This and (4.6) imply that

$$
\inf _{\kappa \in R^{L(2)}}\left\langle-h_{x}(\bar{x}(T))^{*} q_{1}, \kappa\right\rangle_{H}=\inf _{\kappa \in R^{L(2)}}\left\langle-q_{1}, h_{x}(\bar{x}(T)) \kappa\right\rangle_{H_{2}}>-\infty,
$$

which contradicts (2.21) and (4.2). 
Proof of Theorem 2.8. The assumption of this theorem is equivalent to

$$
\left(\bigcap_{j \in \mathcal{I}_{g}} Q_{j}\right) \bigcap \Theta \bigcap \operatorname{cl} R^{L(2)}=\emptyset .
$$

Then, by the same arguments as in the proof of Theorem 2.5, we can get the conclusion.

Proof of Theorem 2.9. By Theorem 2.5, we only need to deal with the case when 0 is on the boundary of $\operatorname{cl} h_{x}(\bar{x}(T))\left(R^{L}\right)$. By the assumption of Theorem 2.9 on $\operatorname{cl} h_{x}(\bar{x}(T))\left(R^{L}\right)$ and the separation theorem, there exists a nonzero $q_{1} \in H_{2}$ such that

$$
\min _{\kappa \in R^{L}}\left\langle q_{1}, h_{x}(\bar{x}(T)) \kappa\right\rangle_{H_{2}}=0
$$

Set $\alpha=0, \beta=q_{1}$ and denote by $p$ the corresponding solution of the adjoint equation (2.2). As before, (4.16) and Lemma 3.5 imply that for a.e. $t \in[0, T]$,

$$
\mathcal{H}(t, \bar{x}(t), u, p(t)) \geq \mathcal{H}(t, \bar{x}(t), \bar{u}(t), p(t)), \quad \forall u \in U
$$

This yields (2.3). Furthermore, if a trajectory-control pair $(w, v)$ of $(2.13)$ satisfies $(2.22)$ with $w_{T}=w(T)$, then

$$
\langle p(T), w(T)\rangle_{H}+\frac{1}{2}\left\langle\beta, h_{x x}(\bar{x}(T))(y(T), y(T))\right\rangle_{H_{2}}=0 .
$$

On the other hand, as in the proof of Theorem 2.5,

$$
\begin{aligned}
& \langle p(T), w(T)\rangle_{H} \\
= & \int_{0}^{T}\left[\mathcal{H}_{u}[t](v(t))+\frac{1}{2}\left(\mathcal{H}_{x x}[t](y(t), y(t))+2 \mathcal{H}_{x u}[t](y(t), u(t))+\mathcal{H}_{u u}[t](u(t), u(t))\right)\right] \mathrm{d} t .
\end{aligned}
$$

Hence,

$$
\begin{aligned}
& \frac{1}{2}\left\langle\beta, h_{x x}(\bar{x}(T))(y(T), y(T))\right\rangle_{H_{2}}+\int_{0}^{T} \mathcal{H}_{u}[t](v(t)) \mathrm{d} t \\
& +\frac{1}{2} \int_{0}^{T}\left(\left\langle\mathcal{H}_{x x}[t] y(t), y(t)\right\rangle_{H}+2 \mathcal{H}_{x u}[t](y(t), u(t))+\mathcal{H}_{u u}[t](u(t), u(t))\right) \mathrm{d} t=0 .
\end{aligned}
$$

This completes the proof.

\section{Two EXAMPLeS}

In this section, we present two illustrative examples for our assumptions to be verified.

\subsection{A controlled hyperbolic equation}

Let $D \subset \mathbb{R}^{3}$ be a bounded domain with a $C^{2}$ boundary $\partial D$. Let $D_{0}$ be a nonempty open subset of $D$. Put

$$
U \triangleq\left\{\left.\hat{u} \in L^{2}(D)|| \hat{u}\right|_{L^{2}(D)} \leq 1\right\}
$$


Consider the following controlled hyperbolic equation:

$$
\begin{cases}\tilde{x}_{t t}(t, \xi)=\Delta \tilde{x}(t, \xi)+\tilde{f}(t, \xi, \tilde{x}(t, \xi))+\chi_{D_{0}}(\xi) \tilde{u}(t, \xi), & (t, \xi) \in(0, T] \times D \\ \tilde{x}(t, \xi)=0, & (t, \xi) \in[0, T] \times \partial D \\ \tilde{x}(0, \xi)=\tilde{x}_{0}(\xi), \quad \tilde{x}_{t}(0, \xi)=\tilde{x}_{1}(\xi), & \xi \in D,\end{cases}
$$

where $\left(\tilde{x}_{0}(\cdot), \tilde{x}_{1}(\cdot)\right) \in H_{0}^{1}(D) \times L^{2}(D)$. The cost functional is

$$
\widehat{J}(\tilde{u})=\int_{D} \tilde{g}_{0}(\xi, \tilde{x}(T, \xi)) \mathrm{d} \xi
$$

and the constraints are

$$
\int_{D} \tilde{g}_{j}(\xi, \tilde{x}(T, \xi)) \mathrm{d} \xi \leq 0, \quad j=1, \ldots, r
$$

and

$$
\int_{D} \tilde{h}_{j}(\xi) \tilde{x}(T, \xi) \mathrm{d} \xi=0, \quad j=1, \ldots, k
$$

Here $\left\{\tilde{h}_{j}\right\}_{j=1}^{k} \subset L^{2}(D)$ are linearly independent. Assume $\tilde{f}$ and $\tilde{g}_{j}(j=0, \ldots, r)$ satisfy the following conditions:

(C1). The function $\tilde{f}:[0, T] \times D \times \mathbb{R} \rightarrow \mathbb{R}$ is Lebesgue measurable in the first two variables, $\tilde{g}_{j}: D \times \mathbb{R} \rightarrow \mathbb{R}$ is Lebesgue measurable in the first variable and $|\tilde{f}(\cdot, \cdot, 0)|_{L^{\infty}((0, T) \times D)}<\infty$.

(C2). The function $r \mapsto \tilde{f}(t, \xi, r)$ (resp. $r \mapsto \tilde{g}_{j}(\xi, r)$ ) has first and second order derivatives, which are also continuous functions in $r$ and are denoted by $\tilde{f}^{\prime}$ and $\tilde{f}^{\prime \prime}\left(\right.$ resp. $\tilde{g}_{j}^{\prime}$ and $\left.\tilde{g}_{j}^{\prime \prime}\right)$. Moreover,

$$
\left|\tilde{f}^{\prime}\right|_{L^{\infty}((0, T) \times D \times \mathbb{R})}+\left|\tilde{f}^{\prime \prime}\right|_{L^{\infty}((0, T) \times D \times \mathbb{R})}+\sum_{j=0}^{r}\left(\left|\tilde{g}_{j}^{\prime}\right|_{L^{\infty}(D \times \mathbb{R})}+\left|\tilde{g}_{j}^{\prime \prime}\right|_{L^{\infty}(D \times \mathbb{R})}\right) \leq C .
$$

Under conditions (C1) and (C2), by the classical well-posedness result for hyperbolic equations (e.g. [16], Sect. 6.1) for each measurable function $\tilde{u}:[0, T] \rightarrow U$, there is a unique weak solution $\tilde{x}(\cdot) \in C^{1}\left([0, T] ; L^{2}(D)\right) \cap$ $C\left([0, T] ; H_{0}^{1}(D)\right)$ of $(5.2)$ such that

$$
|\tilde{x}(\cdot)|_{C^{1}\left([0, T] ; L^{2}(D)\right)}+|\tilde{x}(\cdot)|_{C\left([0, T] ; H_{0}^{1}(D)\right)} \leq C\left(\left|\left(\tilde{x}_{0}, \tilde{x}_{1}\right)\right|_{H_{0}^{1}(D) \times L^{2}(D)}+|\tilde{u}|_{L^{2}\left(0, T ; L^{2}(D)\right)}\right) .
$$

Put $H=H_{0}^{1}(D) \times L^{2}(D), H_{1}=L^{2}(D)$. Then $x=(\tilde{x}, \tilde{\tilde{x}}) \in H$ with $\tilde{x} \in H_{0}^{1}(D)$ and $\tilde{\tilde{x}} \in L^{2}(D)$. Let

$$
D(A)=\left(H^{2}(D) \cap H_{0}^{1}(D)\right) \times H_{0}^{1}(D), \quad A(\tilde{x}, \tilde{\tilde{x}})=(\tilde{\tilde{x}}, \Delta \tilde{x}), \quad \forall(\tilde{x}, \tilde{\tilde{x}}) \in D(A)
$$


and

$$
\left\{\begin{array}{l}
\left.f(t, x(\cdot), u)=(0, \tilde{f}(t, \cdot) \tilde{x}(\cdot))+\chi_{D_{0}}(\cdot) u(\cdot)\right), \quad \forall x=(\tilde{x}, \tilde{\tilde{x}}) \in H, u \in U, \\
g_{0}(x)=\int_{D} \tilde{g}_{0}(\xi, \tilde{x}(\xi)) \mathrm{d} \xi, \quad g_{j}(x)=\int_{D} \tilde{g}_{j}(\xi, \tilde{x}(\xi)) \mathrm{d} \xi, \quad j=1, \ldots, r, \quad \forall x=(\tilde{x}, \tilde{\tilde{x}}) \in H, \\
h(x)=\left(\int_{D} \tilde{h}_{1}(\xi) \tilde{x}(\xi) \mathrm{d} \xi, \ldots, \int_{D} \tilde{h}_{k}(\xi) \tilde{x}(\xi) \mathrm{d} \xi\right)^{\top} \text { for all } x=(\tilde{x}, \tilde{\tilde{x}}) \in H .
\end{array}\right.
$$

By (C1) and (C2), it is clear that (H1) and (H2) hold. Now we prove that $f, h$ and $g_{j}(j=0, \ldots, r)$ are twice continuously Fréchet differentiable. For any $\left(\delta x_{0}(\cdot), \delta x_{1}(\cdot)\right) \in H_{0}^{1}(D) \times L^{2}(D)$, by the Sobolev inequality (e.g. [6], p. 284, Thm. 6), we have that

$$
\begin{aligned}
& \left|\tilde{f}\left(t, \cdot \tilde{x}(\cdot)+\delta x_{0}(\cdot)\right)-\tilde{f}(t, \cdot, \tilde{x}(\cdot))-\tilde{f}^{\prime}(t, \cdot, \tilde{x}(\cdot)) \delta x_{0}(\cdot)\right|_{L^{2}(D)} \\
= & \left|\int_{0}^{1} \tilde{f}^{\prime}\left(t, \cdot \tilde{x}(\cdot)+\theta \delta x_{0}(\cdot)\right) \mathrm{d} \theta \delta x_{0}(\cdot)-\tilde{f}^{\prime}(t, \cdot, \tilde{x}(\cdot)) \delta x_{0}(\cdot)\right|_{L^{2}(D)} \\
= & \left|\int_{0}^{1}\left[\tilde{f}^{\prime}\left(t, \cdot, \tilde{x}(\cdot)+\theta \delta x_{0}(\cdot)\right)-\tilde{f}^{\prime}(t, \cdot, \tilde{x}(\cdot))\right] \mathrm{d} \theta \delta x_{0}(\cdot)\right|_{L^{2}(D)} \\
\leq & \left|\int_{0}^{1}\left[\tilde{f}^{\prime}\left(t, \cdot \tilde{x}(\cdot)+\theta \delta x_{0}(\cdot)\right)-\tilde{f}^{\prime}(t, \cdot, \tilde{x}(\cdot))\right] \mathrm{d} \theta\right|_{L^{3}(D)}\left|\delta x_{0}(\cdot)\right|_{L^{6}(D)} \\
\leq & C\left|\int_{0}^{1}\left[\tilde{f}^{\prime}\left(t, \cdot \tilde{x}(\cdot)+\theta \delta x_{0}(\cdot)\right)-\tilde{f}^{\prime}(t, \cdot \tilde{x}(\cdot))\right] \mathrm{d} \theta\right|_{L^{3}(D)}\left|\delta x_{0}(\cdot)\right|_{H_{0}^{1}(D)} .
\end{aligned}
$$

This implies that

$$
\begin{aligned}
& \lim _{\left|\delta x_{0}\right|_{H_{0}^{1}(D)} \rightarrow 0} \frac{\left|\tilde{f}\left(t, \cdot, \tilde{x}(\cdot)+\delta x_{0}(\cdot)\right)-\tilde{f}(t, \cdot, \tilde{x}(\cdot))-\tilde{f}^{\prime}(t, \cdot, \tilde{x}(\cdot)) \delta x_{0}(\cdot)\right|_{L^{2}(D)}}{\left|\delta x_{0}\right|_{H_{0}^{1}(D)}} \\
& \leq C \lim _{\left|\delta x_{0}\right|_{H_{0}^{1}(D)} \rightarrow 0}\left|\int_{0}^{1}\left[\tilde{f}^{\prime}\left(t, \cdot, \tilde{x}(\cdot)+\theta \delta x_{0}(\cdot)\right)-\tilde{f}^{\prime}(t, \cdot, \tilde{x}(\cdot))\right] \mathrm{d} \theta\right|_{L^{3}(D)}=0 .
\end{aligned}
$$

Thus, for any $x \in H$ and $u \in U$, it holds that for any $\left(\delta x_{0}(\cdot), \delta x_{1}(\cdot)\right) \in H_{0}^{1}(D) \times L^{2}(D)$ (recall (5.6) for the definition of $f$ ),

$$
f_{x}(t, x, u)\left(\delta x_{0}(\cdot), \delta x_{1}(\cdot)\right)=\left(0, \tilde{f}^{\prime}(t, \cdot, \tilde{x}(\cdot)) \delta x_{0}(\cdot)\right)
$$

and

$$
\left|f_{x}(t, x, u)\right|_{\mathcal{L}(H)} \leq\left|\tilde{f}^{\prime}(t, \cdot, \tilde{x}(\cdot))\right|_{L^{3}(D)} \leq C \text { for a.e. } t \in[0, T] \text { and for all }(x, u) \in H \times U
$$

Next, for any $\left(\delta x_{0}(\cdot), \delta x_{1}(\cdot)\right),\left(\widetilde{\delta x}_{0}(\cdot), \widetilde{\delta x}_{1}(\cdot)\right) \in H_{0}^{1}(D) \times L^{2}(D)$, we have that

$$
\begin{aligned}
& \left|\tilde{f}^{\prime}\left(t, \cdot, \tilde{x}(\cdot)+\delta x_{0}(\cdot)\right){\widetilde{\delta x_{0}}}_{(\cdot)}-\tilde{f}^{\prime}(t, \cdot, \tilde{x}(\cdot)){\widetilde{\delta x_{0}}}_{(\cdot)}-\tilde{f}^{\prime \prime}(t, \cdot, \tilde{x}(\cdot)) \delta x_{0}(\cdot){\widetilde{\delta x_{0}}}_{(\cdot)}\right|_{L^{2}(D)} \\
\leq & \left|\tilde{f}^{\prime}\left(t, \cdot, \tilde{x}(\cdot)+\delta x_{0}(\cdot)\right)-\tilde{f}^{\prime}(t, \cdot, \tilde{x}(\cdot))-\tilde{f}^{\prime \prime}(t, \cdot, \tilde{x}(\cdot)) \delta x_{0}(\cdot)\right|_{L^{3}(D)}\left|\widetilde{\delta x_{0}}(\cdot)\right|_{L^{6}(D)} \\
= & \left|\int_{0}^{1} \tilde{f}^{\prime \prime}\left(t, \cdot, \tilde{x}(\cdot)+\theta \delta x_{0}(\cdot)\right) \mathrm{d} \theta \delta x_{0}(\cdot)-\tilde{f}^{\prime \prime}(t, \cdot, \tilde{x}(\cdot)) \delta x_{0}(\cdot)\right|_{L^{3}(D)}\left|\widetilde{\delta x_{0}}(\cdot)\right|_{L^{6}(D)}
\end{aligned}
$$




$$
\begin{aligned}
& =\left|\int_{0}^{1}\left[\tilde{f}^{\prime \prime}\left(t, \cdot, \tilde{x}(\cdot)+\theta \delta x_{0}(\cdot)\right)-\tilde{f}^{\prime \prime}(t, \cdot, \tilde{x}(\cdot))\right] \mathrm{d} \theta \delta x_{0}(\cdot)\right|_{L^{3}(D)}\left|{\widetilde{\delta x_{0}}}_{0}(\cdot)\right|_{L^{6}(D)} \\
& \leq\left|\int_{0}^{1}\left[\tilde{f}^{\prime \prime}\left(t, \cdot, \tilde{x}(\cdot)+\theta \delta x_{0}(\cdot)\right)-\tilde{f}^{\prime \prime}(t, \cdot \tilde{x}(\cdot))\right] \mathrm{d} \theta\right|_{L^{6}(D)}\left|\delta x_{0}(\cdot)\right|_{L^{6}(D)}\left|\widetilde{\delta x_{0}}(\cdot)\right|_{L^{6}(D)} \\
& \leq C\left|\int_{0}^{1}\left[\tilde{f}^{\prime \prime}\left(t, \cdot, \tilde{x}(\cdot)+\theta \delta x_{0}(\cdot)\right)-\tilde{f}^{\prime \prime}(t, \cdot, \tilde{x}(\cdot))\right] \mathrm{d} \theta\right|_{L^{6}(D)}\left|\delta x_{0}(\cdot)\right|_{H_{0}^{1}(D)}\left|\widetilde{\delta x_{0}}(\cdot)\right|_{H_{0}^{1}(D)}
\end{aligned}
$$

This implies that

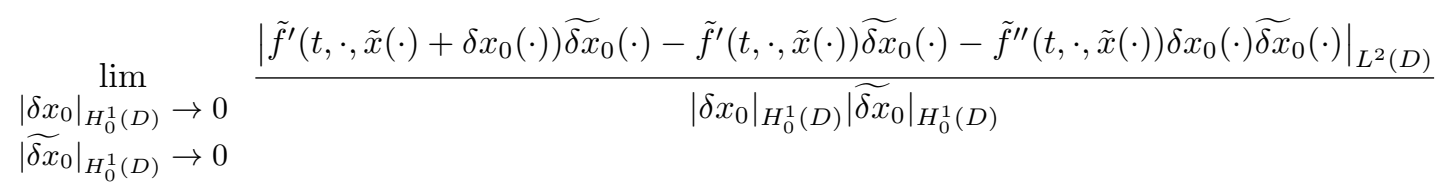

$$
\begin{aligned}
& \leq C \lim _{\left|\delta x_{0}\right|_{H_{0}^{1}(D)} \rightarrow 0}\left|\int_{0}^{1}\left[\tilde{f}^{\prime \prime}(t, \cdot, \tilde{x}(\cdot)+\theta \delta x(\cdot))-\tilde{f}^{\prime \prime}(t, \cdot, \tilde{x}(\cdot))\right] \mathrm{d} \theta\right|_{L^{6}(D)}=0 .
\end{aligned}
$$

Thus, it holds that for any $\left(\delta x_{0}(\cdot), \delta x_{1}(\cdot)\right),\left(\widetilde{\delta x_{0}}(\cdot), \widetilde{\delta x_{1}}(\cdot)\right) \in H_{0}^{1}(D) \times L^{2}(D)$,

$$
f_{x x}(t, x, u)\left(\left(\delta x_{0}, \delta x_{1}\right),\left(\widetilde{\delta x_{0}}, \widetilde{\delta x_{1}}\right)\right)=\left(0, \tilde{f}^{\prime \prime}(t, \cdot, \tilde{x}(\cdot)) \delta x_{0}(\cdot) \widetilde{\delta x_{0}}(\cdot)\right)
$$

and

$$
\left|f_{x x}(t, x, u)\right|_{\mathcal{L}(H, H ; H)} \leq\left|\tilde{f}^{\prime \prime}(t, \cdot, \tilde{x}(\cdot))\right|_{L^{6}(D)} \leq C \text { for a.e. } t \in[0, T] \text { and for all }(x, u) \in H \times U \text {. }
$$

Similarly, we can prove that (recall (5.6) for the definition of $g_{j}(j=0, \ldots, r)$ and $h$ )

$$
\left|g_{j, x}(x)\right|_{H}+\left|g_{j, x x}(x)\right|_{\mathcal{L}(H)} \leq C \text { for all } x \in H \text { and } j=0, \ldots, r
$$

and

$$
\left|h_{x}(x)\right|_{\mathcal{L}\left(H ; \mathbb{R}^{k}\right)}+\left|h_{x x}(x)\right|_{\mathcal{L}\left(H, H ; \mathbb{R}^{k}\right)} \leq C \text { for all } x \in H
$$

Let $\left(\left(\overline{\tilde{x}}, \overline{\tilde{x}}_{t}\right), \overline{\tilde{u}}\right)$ be a local minimizer. By $(5.1)$, we know that $U$ is the unit ball in $L^{2}(D)$. Then

$$
T_{U}^{b}(\overline{\tilde{u}}(t))= \begin{cases}\left\{v \in L^{2}(D) \mid\langle v, \overline{\tilde{u}}(t)\rangle_{L^{2}(D)} \leq 0\right\} & \text { if }|\overline{\tilde{u}}(t)|_{L^{2}(D)}=1 \\ L^{2}(D) & \text { otherwise. }\end{cases}
$$

Consider a critical pair $\left(\left(\tilde{y}, \tilde{y}_{t}\right), \tilde{u}\right) \in \mathcal{C}\left(\left(\overline{\tilde{x}}, \overline{\tilde{x}}_{t}\right), \overline{\tilde{u}}\right)$. Then $\tilde{u}(t) \in T_{U}^{b}(\overline{\tilde{u}}(t))$ for a.e. $t \in[0, T]$. Assume in addition that $\tilde{u} \in L^{\infty}\left(0, T ; L^{2}(D)\right)$ and that for some $\delta \in(0,1)$ and a.e. $t \in[0, T]$,

$$
|\overline{\tilde{u}}(t)|_{L^{2}(D)}>1-\delta \Longrightarrow\langle\overline{\tilde{u}}(t), \tilde{u}(t)\rangle_{L^{2}(D)} \leq 0 .
$$

Define $M:=\operatorname{ess}_{\sup } \operatorname{se[0,T]}_{\mid}|\tilde{u}(t)|_{L^{2}(D)}$ and observe that for a.e. $t \in[0, T]$ such that $|\overline{\tilde{u}}(t)|_{L^{2}(D)} \leq 1-\delta$ and for every $\varepsilon \in[0, \delta / M]$ we have $|\overline{\tilde{u}}(t)+\varepsilon \tilde{u}(t)|_{L^{2}(D)} \leq 1$. Therefore $\operatorname{dist}(\overline{\tilde{u}}(t)+\varepsilon \tilde{u}(t), U)=0$ and $0 \in T_{U}^{b(2)}(\overline{\tilde{u}}(t), \tilde{u}(t))$. 
Consider next $t \in[0, T]$ such that $|\overline{\tilde{u}}(t)|_{L^{2}(D)}>1-\delta$. Then for all small $\varepsilon>0$ we have

$$
\begin{aligned}
& \left.\left.\left|\overline{\tilde{u}}(t)+\varepsilon \tilde{u}(t)-\varepsilon^{2}\right| \tilde{u}(t)\right|_{L^{2}(D)} ^{2} \overline{\tilde{u}}(t)\right|_{L^{2}(D)} ^{2} \\
& =\left|\left(1-\varepsilon^{2}|\tilde{u}(t)|_{L^{2}(D)}^{2}\right) \overline{\tilde{u}}(t)+\varepsilon \tilde{u}(t)\right|_{L^{2}(D)}^{2} \\
& \leq\left(1-\varepsilon^{2}|\tilde{u}(t)|_{L^{2}(D)}^{2}\right)^{2}|\overline{\tilde{u}}(t)|_{L^{2}(D)}^{2}+\varepsilon^{2}|\tilde{u}(t)|_{L^{2}(D)}^{2} \\
& \leq\left(1-\varepsilon^{2}|\tilde{u}(t)|_{L^{2}(D)}^{2}\right)^{2}+\varepsilon^{2}|\tilde{u}(t)|_{L^{2}(D)}^{2} \\
& =1-\varepsilon^{2}|\tilde{u}(t)|_{L^{2}(D)}^{2}+\varepsilon^{4}|\tilde{u}(t)|_{L^{2}(D)}^{2} M^{2}<1 .
\end{aligned}
$$

Consequently, $\operatorname{dist}(\overline{\tilde{u}}(t)+\varepsilon \tilde{u}(t), U) \leq \varepsilon^{2}|\tilde{u}(t)|_{L^{2}(D)}^{2}|\overline{\tilde{u}}(t)|_{L^{2}(D)}$ whenever $\varepsilon>0$ is sufficiently small, and $-|\tilde{u}(t)|_{L^{2}(D)}^{2} \overline{\tilde{u}}(t) \in T_{U}^{b(2)}(\overline{\tilde{u}}(t), \tilde{u}(t))$. This implies that $V^{2}(\overline{\tilde{u}}, \tilde{u}) \cap L^{2}\left(0, T ; H_{1}\right) \neq \emptyset$.

In the setting of this subsection, the control system (2.13) reads

$$
\left\{\begin{array}{rlrl}
\tilde{w}_{t t}(t, \xi)=\Delta \tilde{w}(t, \xi)+\tilde{f}^{\prime}(t, \xi, \overline{\tilde{x}}(\xi)) \tilde{w}(t, \xi) & \\
& +\frac{1}{2} \tilde{f}^{\prime \prime}(t, \xi, \tilde{x}(\xi)) \tilde{y}(t, \xi)^{2}+\chi_{D_{0}}(t, \xi) \tilde{v}(t, \xi), & & (t, \xi) \in(0, T] \times D, \\
\tilde{w}(t, \xi)=0, & & (t, \xi) \in(0, T] \times \partial D, \\
\tilde{w}(0, \xi)=0, & \tilde{w}_{t}(0, \xi)=0, & \xi \in D .
\end{array}\right.
$$

Here $\tilde{v}(t) \in T_{U}^{b(2)}(\overline{\tilde{u}}(t), \tilde{u}(t))$ for a.e. $t \in[0, T]$ with $\tilde{v}(\cdot) \in L^{2}\left(0, T ; L^{2}(D)\right)$ and $\tilde{y}$ solves

$$
\begin{cases}\tilde{y}_{t t}(t, \xi)=\Delta \tilde{y}(t, \xi)+\tilde{f}^{\prime}(t, \xi, \overline{\tilde{x}}(\xi)) \tilde{y}(t, \xi)+\chi_{D_{0}}(t, \xi) \tilde{u}(t, \xi), & (t, \xi) \in(0, T] \times D, \\ \tilde{y}(t, \xi)=0, & (t, \xi) \in(0, T] \times \partial D, \\ \tilde{y}(0, \xi)=0, \tilde{y}_{t}(0, \xi)=0, & \xi \in D .\end{cases}
$$

From (5.5), we know that $\Theta$ is a finite codimensional subspace of $H$. Let us consider two different cases:

Case $1 R^{L(2)} \subset \Theta$. In this case, $\widetilde{\Theta}=\Theta$ fulfills $(\mathbf{H} 4)$.

Case $2 \emptyset \neq R^{L(2)} \varsubsetneqq \Theta$. Denote by $\mathbf{P}_{\Theta}$ the orthogonal projection operator from $H$ to $\Theta$ and by $I$ the identity operator on $H$. Then we have that $\left(I-\mathbf{P}_{\Theta}\right) R^{L(2)} \neq\{0\}$. Since $\Theta$ is a finite codimensional subspace of $H$, there are only finitely many linearly independent elements in $\left(I-\mathbf{P}_{\Theta}\right) R^{L(2)}$. Denote by $\left\{r_{j}\right\}_{j=1}^{k_{1}}$ these linearly independent elements. Then we know that $\left(I-\mathbf{P}_{\Theta}\right) R^{L(2)} \subset H_{3} \triangleq \operatorname{span}\left\{r_{1}, \ldots, r_{k_{1}}\right\}$. Then $\Theta \oplus H_{3}$ is a closed subspace of $H, \widetilde{\Theta} \subset \Theta \oplus H_{3}$ and $\operatorname{int}_{\Theta \oplus H_{3}} \widetilde{\Theta} \neq \emptyset$. By choosing $\widetilde{H}=\Theta \oplus H_{3}$, we get (H4).

\subsection{A controlled parabolic equation}

Consider the following controlled parabolic equation:

$$
\begin{cases}\hat{x}_{t}(t, \xi)=\Delta \hat{x}(t, \xi)+\hat{f}(t, \xi, \hat{x}(t, \xi))+\chi_{D_{0}}(\xi) \hat{u}(t, \xi), & (t, \xi) \in(0, T] \times D, \\ \hat{x}(t, \xi)=0, & (t, \xi) \in(0, T] \times \partial D, \\ \hat{x}(0, \xi)=\hat{x}_{0}(\xi), & \xi \in D,\end{cases}
$$


where $\hat{x}_{0}(\cdot) \in L^{2}(D)$, and $\hat{u}(t, \cdot) \in U$ (recall (5.1) for the definition of $U$ ) for a.e. $t \in[0, T]$ and is measurable with respect to $t$. The cost functional is

$$
\widehat{J}(\hat{u})=\int_{D} \hat{g}_{0}(\xi, \hat{x}(T, \xi)) \mathrm{d} \xi
$$

and the constraints are (5.4) and (5.5) with linearly independent $\left\{\tilde{h}_{j}\right\}_{j=1}^{k} \subset L^{2}(D)$ and $\tilde{x}$ replaced by $\hat{x}$. Assume $\hat{f}$ and $\hat{g}_{j}(j=0, \ldots, r)$ satisfy the following conditions:

(C3). The function $\hat{f}:[0, T] \times D \times \mathbb{R} \rightarrow \mathbb{R}$ is Lebesgue measurable in the first two variables, $\hat{g}_{j}: D \times \mathbb{R} \rightarrow \mathbb{R}$ $(j=0, \ldots, r)$ is Lebesgue measurable in the first variable and $|\tilde{f}(\cdot, \cdot, 0)|_{L^{\infty}((0, T) \times D)}<\infty$.

(C4). The function $r \mapsto \hat{f}(t, \xi, r)\left(r e s p . r \mapsto \hat{g}_{j}(\xi, r)\right)$ is twice continuously differentiable. Denote by $\hat{f}^{\prime}$ and $\hat{f}^{\prime \prime}\left(\right.$ resp. $\hat{g}_{j}^{\prime}$ and $\left.\hat{g}_{j}^{\prime \prime}\right)$ the first and second order derivatives, respectively. Moreover, assume that

$$
\left|\hat{f}^{\prime}\right|_{L^{\infty}((0, T) \times D \times \mathbb{R})}+\left|\hat{f}^{\prime \prime}\right|_{L^{\infty}((0, T) \times D \times \mathbb{R})}+\sum_{j=0}^{r}\left(\left|\hat{g}_{j}^{\prime}\right|_{L^{\infty}(D \times \mathbb{R})}+\left|\hat{g}_{j}^{\prime \prime}\right|_{L^{\infty}(D \times \mathbb{R})}\right) \leq C .
$$

Under conditions (C3) and (C4), by the classical well-posedness result for parabolic equations (e.g. [6], p. 535, Exam. 1) for each Lebesgue measurable function $\hat{u}:[0, T] \rightarrow U$, there is a unique weak solution $\hat{x}(\cdot) \in$ $C\left([0, T] ; L^{2}(D)\right) \cap L^{2}\left(0, T ; H_{0}^{1}(D)\right)$ of $(5.9)$ such that

$$
|\hat{x}(\cdot)|_{C\left([0, T] ; L^{2}(D)\right)}+|\hat{x}(\cdot)|_{L^{2}\left(0, T ; H_{0}^{1}(D)\right)} \leq C\left(\left|\hat{x}_{0}\right|_{L^{2}(D)}+|\hat{u}|_{L^{2}\left(0, T ; L^{2}(D)\right)}\right) .
$$

Put $H=L^{2}(D), H_{1}=L^{2}(D), X=L^{6}(D)$. It is well known that $|\cdot|_{X}$ is Fréchet differentiable away from zero. Let

$$
\left\{\begin{array}{l}
f(t, x, u)=\hat{f}(t, \cdot, x(\cdot))+\chi_{D_{0}}(\cdot) u(\cdot), \quad \forall x \in H, \quad u \in U, \\
g_{0}(x)=\int_{D} \hat{g}_{0}(\xi, x(\xi)) \mathrm{d} \xi, \quad g_{j}(x)=\int_{D} \hat{g}_{j}(\xi, x(\xi)) \mathrm{d} \xi, \quad j=1, \ldots, r, \quad \forall x \in H, \\
h(x)=\left(\int_{D} \tilde{h}_{1}(\xi) x(\xi) \mathrm{d} \xi, \ldots, \int_{D} \tilde{h}_{k}(\xi) x(\xi) \mathrm{d} \xi\right)^{\top} \text { for all } x \in H .
\end{array}\right.
$$

By the smoothing effect of the $C_{0}$-semigroup generated by the homogeneous Dirichlet Laplacian, the Sobolev embedding theorem (e.g. [6], p. 284, Thm. 6) and (5.11), we get (S1).

By (C3) and (C4), it is clear that (S2) holds. Now we prove (S3). For any $\delta x(\cdot) \in L^{6}(D)$, we have that

$$
\begin{aligned}
& \left|\hat{f}(t, \cdot, \hat{x}(\cdot)+\delta x(\cdot))-\hat{f}(t, \cdot, \hat{x}(\cdot))-\hat{f}^{\prime}(t, \cdot, \hat{x}(\cdot)) \delta x(\cdot)\right|_{L^{2}(D)} \\
= & \left|\int_{0}^{1} \hat{f}^{\prime}(t, \cdot, \hat{x}(\cdot)+\theta \delta x(\cdot)) \mathrm{d} \theta \delta x(\cdot)-\hat{f}^{\prime}(t, \cdot, \hat{x}(\cdot)) \delta x(\cdot)\right|_{L^{2}(D)} \\
= & \left|\int_{0}^{1}\left[\hat{f}^{\prime}(t, \cdot, \hat{x}(\cdot)+\theta \delta x(\cdot))-\hat{f}^{\prime}(t, \cdot, \hat{x}(\cdot))\right] \mathrm{d} \theta \delta x(\cdot)\right|_{L^{2}(D)} \\
\leq & \left|\int_{0}^{1}\left[\hat{f}^{\prime}(t, \cdot, \hat{x}(\cdot)+\theta \delta x(\cdot))-\hat{f}^{\prime}(t, \cdot, \hat{x}(\cdot))\right] \mathrm{d} \theta\right|_{L^{3}(D)}|\delta x(\cdot)|_{L^{6}(D)}
\end{aligned}
$$


This implies that

$$
\begin{aligned}
& \lim _{|\delta x|_{L^{6}(D)} \rightarrow 0} \frac{\left|\hat{f}(t, \cdot, \hat{x}(\cdot)+\delta x(\cdot))-\hat{f}(t, \cdot \hat{x}(\cdot))-\hat{f}^{\prime}(t, \cdot, \hat{x}(\cdot)) \delta x(\cdot)\right|_{L^{2}(D)}}{|\delta x|_{L^{6}(D)}} \\
& \leq \lim _{|\delta x|_{L^{6}(D)} \rightarrow 0}\left|\int_{0}^{1}\left[\hat{f}^{\prime}(t, \cdot, \hat{x}(\cdot)+\theta \delta x(\cdot))-\hat{f}^{\prime}(t, \cdot, \hat{x}(\cdot))\right] \mathrm{d} \theta\right|_{L^{3}(D)}=0 .
\end{aligned}
$$

Thus, for any $\delta x(\cdot) \in L^{6}(D)$ and $(x, u) \in X \times U$, from (5.12), we see that

$$
f_{x}(t, x, u) \delta x=\hat{f}^{\prime}(t, \cdot, x(\cdot)) \delta x(\cdot)
$$

and

$$
\left|f_{x}(t, x, u)\right|_{\mathcal{L}(X ; H)} \leq\left|\hat{f}^{\prime}(t, \cdot, x(\cdot))\right|_{L^{3}(D)} \leq C \text { for a.e. } t \in[0, T] \text { and for all }(x, u) \in X \times U
$$

Next, for any $\delta x(\cdot), \widetilde{\delta x}(\cdot) \in L^{6}(D)$, we have that

$$
\begin{aligned}
& \left|\hat{f}^{\prime}(t, \cdot, \hat{x}(\cdot)+\delta x(\cdot)) \widetilde{\delta x}(\cdot)-\hat{f}^{\prime}(t, \cdot \hat{x}(\cdot)) \widetilde{\delta x}(\cdot)-\hat{f}^{\prime \prime}(t, \cdot, \hat{x}(\cdot)) \delta x(\cdot) \widetilde{\delta x}(\cdot)\right|_{L^{2}(D)} \\
\leq & \left|\hat{f}^{\prime}(t, \cdot, \hat{x}(\cdot)+\delta x(\cdot))-\hat{f}^{\prime}(t, \cdot, \hat{x}(\cdot))-\hat{f}^{\prime \prime}(t, \cdot, \hat{x}(\cdot)) \delta x(\cdot)\right|_{L^{3}(D)}|\widetilde{\delta x}(\cdot)|_{L^{6}(D)} \\
= & \left|\int_{0}^{1} \hat{f}^{\prime \prime}(t, \cdot, \hat{x}(\cdot)+\theta \delta x(\cdot)) \mathrm{d} \theta \delta x(\cdot)-\hat{f}^{\prime \prime}(t, \cdot, \hat{x}(\cdot)) \delta x(\cdot)\right|_{L^{3}(D)}|\widetilde{\delta x}(\cdot)|_{L^{6}(D)} \\
= & \left|\int_{0}^{1}\left[\hat{f}^{\prime \prime}(t, \cdot, \hat{x}(\cdot)+\theta \delta x(\cdot))-\hat{f}^{\prime \prime}(t, \cdot, \hat{x}(\cdot))\right] \mathrm{d} \theta \delta x(\cdot)\right|_{L^{3}(D)}|\widetilde{\delta x}(\cdot)|_{L^{6}(D)} \\
\leq & \left|\int_{0}^{1}\left[\hat{f}^{\prime \prime}(t, \cdot, \hat{x}(\cdot)+\theta \delta x(\cdot))-\hat{f}^{\prime \prime}(t, \cdot, \hat{x}(\cdot))\right] \mathrm{d} \theta\right|_{L^{6}(D)}|\delta x(\cdot)|_{L^{6}(D)}|\widetilde{\delta x}(\cdot)|_{L^{6}(D)}
\end{aligned}
$$

This implies that

$$
\begin{aligned}
& \quad \lim _{|\delta x|_{L^{6}(D)} \rightarrow 0} \frac{\left|\hat{f}^{\prime}(t, \cdot, \hat{x}(\cdot)+\delta x(\cdot)) \widetilde{\delta x}(\cdot)-\hat{f}^{\prime}(t, \cdot, \hat{x}(\cdot)) \widetilde{\delta x}(\cdot)-\hat{f}^{\prime \prime}(t, \cdot, \hat{x}(\cdot)) \delta x(\cdot) \widetilde{\delta x}(\cdot)\right|_{L^{2}(D)}}{|\delta x|_{L^{6}(D)}|\widetilde{\delta x}|_{L^{6}(D)}} \\
& \leq \lim _{|\delta x|_{L^{6}(D)} \rightarrow 0}\left|\int_{0}^{1}\left[\hat{f}^{\prime \prime}(t, \cdot, \hat{x}(\cdot)+\theta \delta x(\cdot))-\hat{f}^{\prime \prime}(t, \cdot, \hat{x}(\cdot))\right] \mathrm{d} \theta\right|_{L^{6}(D)}=0 .
\end{aligned}
$$

Consequently, for any $\delta x(\cdot), \widetilde{\delta x}(\cdot) \in L^{6}(D)$ and $(x, u) \in X \times U$,

$$
f_{x x}(t, x, u)(\delta x, \widetilde{\delta x})=\hat{f}^{\prime \prime}(t, \cdot, x(\cdot)) \delta x(\cdot) \widetilde{\delta x}(\cdot)
$$

and

$$
\left|f_{x x}(t, x, u)\right|_{\mathcal{L}(X, X ; H)} \leq\left|\hat{f}^{\prime \prime}(t, \cdot, x(\cdot))\right|_{L^{6}(D)} \leq C \text { for a.e. } t \in[0, T] \text { and for all }(x, u) \in X \times U .
$$

Similarly, we can prove that

$$
\left|g_{j, x}(x)\right|_{H}+\left|g_{j, x x}(x)\right|_{\mathcal{L}(X ; H)} \leq C \text { for all } x \in X, \text { for } j=0, \ldots, r
$$


and

$$
\left|h_{x}(x)\right|_{\mathcal{L}\left(X ; \mathbb{R}^{k}\right)}+\left|h_{x x}(x)\right|_{\mathcal{L}\left(X, X ; \mathbb{R}^{k}\right)} \leq C \text { for all } x \in X
$$

Next, similarly to the proof in Section 5.1, we can show that (S5) holds. As before, if $\tilde{u} \in L^{\infty}\left(0, T ; L^{2}(D)\right)$ is such that $\tilde{u}(t) \in T_{U}^{b}(\overline{\tilde{u}}(t))$ for a.e. $t \in[0, T]$ and for some $\delta>0$ and a.e. $t \in[0, T]$

$$
|\overline{\tilde{u}}(t)|_{L^{2}(D)}>1-\delta \Longrightarrow\langle\overline{\tilde{u}}(t), \tilde{u}(t)\rangle_{L^{2}(D)} \leq 0
$$

then $V^{2}(\bar{u}, \tilde{u}) \cap L^{2}\left(0, T ; H_{1}\right) \neq \emptyset$.

\section{REFERENCES}

[1] J.-P. Aubin and H. Frankowska, Set-valued analysis. Springer Science \& Business Media (2009).

[2] P. Cannarsa, G. Da Prato and H. Frankowska, Domain invariance for local solutions of semilinear evolution equations in Hilbert spaces. J. London Math. Soc. 102 (2020) 287-318.

[3] E. Casas and M. Mateos, Second order optimality conditions for semilinear elliptic control problems with finitely many state constraints. SIAM J. Control Optim. 40 (2002) 1431-1454.

[4] E. Casas and F. Tröltzsch, Second-order necessary and sufficient optimality conditions for optimization problems and applications to control theory. SIAM J. Control Optim. 13 (2002) 406-431.

[5] E. Casas and F. Tröltzsch, Second order optimality conditions and their role in PDE control. Jahresber. Dtsch. Math.-Ver. 117 (2015) 3-44.

[6] L.C. Evans, Partial differential equations. American Mathematical Society, Providence, RI (2010).

[7] H. Frankowska, High order inverse function theorems. Ann. Inst. H. Poincaré Anal. Non Linéaire 6 (1989) $283-303$.

[8] H. Frankowska, On second-order necessary conditions in optimal control of problems with mixed final point constraints. In Proceedings of 58th IEEE Conference on Decision and Control, Nice, France, December 11-13 (2019), DOI: 10.1109/CDC40024.2019.9029546.

[9] H. Frankowska, D. Hoehener and D. Tonon, A second-order maximum principle in optimal control under state constraints. Serdica Math. J. 39 (2013) 233-270.

[10] H. Frankowska and N.P. Osmolovskii, Second-order necessary conditions for a strong local minimum in a control problem with general control constraints. Appl. Math. Optim. 80 (2019) 135-164.

[11] H. Frankowska and N.P. Osmolovskii, Distance estimates to feasible controls for systems with final point constraints and second order necessary optimality conditions. Syst. Control Lett. 144 (2020) 104770.

[12] H. Frankowska, H. Zhang and X. Zhang, Necessary optimality conditions for local minimizers of stochastic optimal control problems with state constraints. Trans. Amer. Math. Soc. 372 (2019) 1289-1331.

[13] X. Li and J. Yong, Optimal control theory for infinite dimensional systems. Birkhäuser Boston, Inc., Boston, MA (1995).

[14] X. Liu, Q. Lü and X. Zhang, Finite codimensional controllability, and optimal control problems with endpoint state constraints. J. Math. Pures Appl. 138 (2020) 164-203.

[15] Q. Lü and X. Zhang, General Pontryagin-type stochastic maximum principle and backward stochastic evolution equations in infinite dimensions. Springer (2014).

[16] A. Pazy, Semigroups of linear operators and applications to partial differential equations. Springer-Verlag, New York (1983).

[17] L.S. Pontryagin, V.G. Boltyanskii, R.V. Gamkrelidze and E.F. Mishchenko, Mathematical theory of optimal processes. Interscience Publishers John Wiley \& Sons, Inc., New York-London (1962).

[18] J.-P. Raymond and F. Tröltzsch, Second order sufficient optimality conditions for nonlinear parabolic control problems with state constraints. Discrete Contin. Dynam. Syst. 6 (2000) 431-450.

[19] A. Rösch and F. Tröltzsch, Sufficient second-order optimality conditions for a parabolic optimal control problem with pointwise control-state constraints. SIAM J. Control Optim. 42 (2003) 138-154.

[20] F. Tröltzsch and D. Wachsmuth, Second-order sufficient optimality conditions for the optimal control of Navier-Stokes equations. ESAIM: COCV 12 (2006) 93-119.

[21] J. Yong and X. Zhou, Stochastic controls. Hamiltonian systems and HJB equations. Springer-Verlag, New York (1999).

[22] E. Zuazua, Controllability and observability of partial differential equations: some results and open problems. Vol. 3 of In Handbook of Differential Equations: Evolutionary Equations. Elsevier Science (2006) 527-621. 